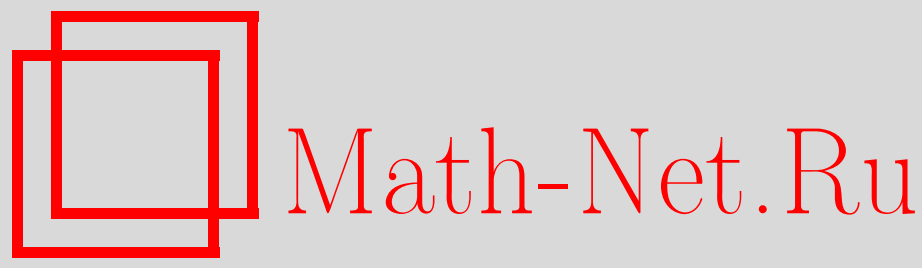

Н. В. Антонов, А. В. Малышев, Влияние сильно анизотропного турбулентного перемешивания на критическое поведение: ренормгрупповой анализ двух необычных систем, ТМФ, 2011, том 167, номер 1, 50-77

DOI: https://doi.org/10.4213/tmf6625

Использование Общероссийского математического портала Math-Net.Ru подразумевает, что вы прочитали и согласны с пользовательским соглашением http://www.mathnet.ru/rus/agreement

Параметры загрузки:

IP : 54.89 .56 .158

26 апреля 2023 г., $12: 35: 41$

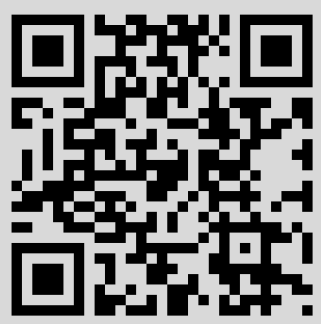




\title{
ВЛИЯНИЕ СИЛЬНО АНИЗОТРОПНОГО ТУРБУЛЕНТНОГО ПЕРЕМЕШИВАНИЯ НА КРИТИЧЕСКОЕ ПОВЕДЕНИЕ: РЕНОРМГРУППОВОЙ АНАЛИЗ ДВУХ НЕОБЫЧНЫХ СИСТЕМ
}

\begin{abstract}
Рассмотрено влияние сильно анизотропного турбулентного перемешивания на критическое поведение двух систем: критической динамики модели $\varphi^{3}$, описыващей универсальные свойства метастабильных состояний вблизи фазового перехода первого рода, и реакционно-диффузионной системы вблизи точки перехода второго рода между флуктуационным и абсорбционным состояниями (простой эпидемический процесс, или процесс Грибова). В обоих случаях продемонстрировано существование нового сильно неравновесного и анизотропного скейлингового режима (класса универсальности), для которого существенны как перемешивание, так и нелинейность по параметру порядка. Соответствующие критические размерности вычислены в однопетлевом приближении ренормализационной группы.
\end{abstract}

Ключевые слова: критическое поведение, турбулентное перемешивание, ренормализационная группа.

\section{1. ВВЕДЕНИЕ}

Многочисленные системы самой разной физической природы (магнетики, системы типа жидкость-пар, бинарные смеси) демонстрируют очень интересное поведение в окрестности критической точки (фазового перехода второго рода). Оказывается, что различные термодинамические величины (теплоемкость, спонтанная намагниченность, восприимчивость и т. д.) имеют степенные особенности. Количественные характеристики такого поведения (например, критические размерности) зависят только от глобальных свойств системы (таких как симметрия или размерность пространства), и поэтому могут быть описаны на основе небольшого числа базовых моделей. Это позволяет объединять системы, различающиеся множеством несущественных (с точки зрения критического поведения) деталей в единые классы, характеризуемые одним и тем же набором критических показателей.

* Научно-исследовательский институт физики им. В. А. Фока, Санкт-Петербургский государственный университет, Санкт-Петербург, Россия. E-mail: antonov@pobox.spbu.ru 
Последовательное количественное описание критического поведения можно дать в рамках метода ренормализационной группы (РГ). В РГ-подходе возможные критические режимы связываются с инфракрасно-устойчивыми (ИК-устойчивыми) неподвижными точками некоторой ренормируемой теоретико-полевой модели. Цель теории - выделить основные классы универсальности, установить существование нужных неподвижных точек для соответствующих моделей, вычислить для них критические размерности и скейлинговые функции в рамках последовательной теории возмущений. Подробное изложение аппарата РГ и его применений в теории критического поведения содержится в монографии [1].

Наиболее типичные фазовые переходы принадлежат классу $O(n)$-симметричной модели $\varphi^{4} n$-компонентного скалярного параметра порядка. Универсальные характеристики такого критического поведения зависят только от $n$ и размерности пространства $d$. Однако в последние годы все больший интерес привлекают фазовые переходы в системах, далеких от состояния термодинамического равновесия (см., например, обзорную статью [2] и цитированную в ней литературу). Их критическое поведение гораздо более многообразно и пока недостаточно хорошо изучено.

Известно, что сильное влияние на критическое поведение могут оказывать внешние возмущения, примеси, гравитация, движение самой среды и т. д. Это может приводить к существенному искажению "идеального" критического поведения, а в некоторых случаях - даже к появлению новых классов универсальности [3]-[9]. Такие в целом разные системы, как, например, магнетик со случайно распределенными примесями или бинарная смесь в турбулентной среде, объединяет общая особенность: существование неравновесных стационарных состояний с новым набором критических показателей. Кроме того, для таких систем при наличии выделенного направления критическое поведение оказывается сильно анизотропным: различным направлениям в пространстве соответствуют разные критические размерности.

Полномасштабная модель критической жидкости, подверженной сильно анизотропному турбулентному перемешиванию, должна описываться с помощью сохраняющегося (бинарная смесь, система жидкость-пар) или несохраняющегося (жидкие кристаллы) параметра порядка, взаимодействующего с полем скорости, которое в свою очередь подчиняется нелинейному динамическому уравнению (например, уравнению Навье-Стокса со случайной силой), и содержать анизотропию, вводимую путем задания начальных или граничных условий.

В настоящей работе мы применяем подход РГ к несколько упрощенным моделям, в которых введено взаимодействие с гауссовым полем скорости, имитирующим некоторые реальные свойства турбулентности. Этого оказывается достаточно, чтобы уловить основную особенность проблемы: существование нового, неравновесного и сильно анизотропного класса универсальности. Мы рассматриваем две динамические модели. Первая из них - модель, описывающая фазовый переход между так называемым абсорбционным (неактивным) и флуктуационным (активным) состояниями некоторой реакционно-диффузионной системы. Более конкретно, мы рассматриваем процесс типа Грибова, называемый так потому, что соответствующая полевая модель близка к хорошо известной реджеонной теории поля [2]. Вторая модель - это релаксационная динамика несохраняющегося скалярного параметра порядка для модели с кубическим взаимодействием типа $\varphi^{3}$. Как обнаружено недавно, эта модель описывает некоторые универсальные свойства метастабильных состояний, возникающих при фазовых переходах первого рода [10]. 
План работы таков. В разделе 2 дано описание моделей в терминах теории поля с соответствующей диаграммной техникой. В разделе 3 проанализированы ультрафиолетовые (УФ) расходимости моделей; показано, что модели являются мультипликативно-ренормируемыми, и приведены ренормированные функционалы действия. Раздел 4 посвящен подсчету констант ренормировки $Z$ для обеих моделей в однопетлевом приближении. В разделе 5 получены дифференциальные уравнения РГ и вычислены $\beta$-функции и аномальные размерности $\gamma$ для всех полей и параметров (в том же приближении). Раздел 6 посвящен анализу неподвижных точек. В разделе 7 приведены результаты для наборов критических размерностей для всех режимов (всех неподвижных точек). Оказывается, что три из четырех неподвижных точек в обеих моделях отвечают уже известным типам критического поведения: свободной теории, пассивному скалярному полю и обычному фазовому переходу без перемешивания. Точки четвертого типа отвечают новым неравновесным классам универсальности, для которых одновременно существенны и нелинейности моделей, и турбулентное перемешивание. В однопетлевом приближении получается, что для заданных значений параметров $\varepsilon$ (отклонение размерности от логарифмической) и $\xi$ (показатель степени в корреляторе поля скорости), по которым идет разложение, ИК-устойчивой является лишь одна из точек, т. е. реализуется определенный критический режим. В этом отношении наши результаты близки к полученным ранее в работах [8], где рассматривалось влияние турбулентного перемешивания на критическое поведение равновесной системы типа $\varphi^{4}$. В качестве частного следствия общих скейлинговых соотношений рассмотрено расплывание облака примесных частиц. Результаты работы кратко суммированы в разделе 8.

В полевой формулировке обе модели выглядят схоже, отличаясь только видом потенциала взаимодействия и добавочным слагаемым в квадратичной части, так что мы будем вести их анализ параллельно. Где это необходимо, результаты для модели с взаимодействием Грибова будут помечаться индексом 1 , результатам для второй модели будет отвечать индекс 2.

\section{2. ТЕОРЕТИКО-ПОЛЕВАЯ ФОРМУЛИРОВКА МОДЕЛИ}

В настоящей работе мы рассматриваем две динамические модели. Обратимся теперь к их более детальному определению и переформулировке на языке теории поля. В непрерывном пределе процесс Грибова (он же направленный процесс протекания, или простой эпидемический процесс) описывается стохастическим дифференциальным уравнением вида [2]

$$
\partial_{t} \varphi(t, \mathbf{x})=\lambda_{0}\left(\partial^{2}-\tau_{0}\right) \varphi(t, \mathbf{x})-\frac{g_{0} \lambda_{0}}{2} \varphi^{2}(t, \mathbf{x})+\sqrt{\varphi(t, \mathbf{x})} \zeta(t, \mathbf{x}),
$$

где $\varphi(t, \mathbf{x})$ - концентрация распространяющегося агента, $\tau \propto\left(p-p_{\mathrm{c}}\right)$ - отклонение вероятности процесса инфицирования (для определенности использована терминология процессов распространения эпидемий) от критического значения, $g_{0}$ - константа взаимодействия, а случайный шум $\zeta(t, \mathbf{x})$ с гауссовым распределением и заданным коррелятором

$$
\left\langle\zeta(t, \mathbf{x}) \zeta\left(t^{\prime}, \mathbf{x}^{\prime}\right)\right\rangle=g_{0} \lambda_{0} \delta\left(t-t^{\prime}\right) \delta^{(d)}\left(\mathbf{x}-\mathbf{x}^{\prime}\right)
$$

моделирует случайные флуктуации. Здесь и далее $d$-размерность пространства векторов $\mathbf{x}$, индекс 0 отмечает различные неренормированные параметры моделей. 
В уравнении (1) множитель $\sqrt{\varphi}$ перед слагаемым, отвечающим шуму, обеспечивает полное исчезновение флуктуаций в неактивной (абсорционной) фазе.

Обратимся ко второй модели. Релаксационную динамику несохраняющегося скалярного параметра порядка $\varphi(t, \mathbf{x})$ будем описывать с помощью стохастического дифференциального уравнения, подобного (1), но с так называемым аддитивным шумом (без дополнительного зависящего от поля $\varphi$ множителя):

$$
\partial_{t} \varphi(t, \mathbf{x})=\lambda_{0}\left(\partial^{2}-\tau_{0}\right) \varphi(t, \mathbf{x})-\frac{g_{0} \lambda_{0}}{2} \varphi^{2}(t, \mathbf{x})+\zeta(t, \mathbf{x}) .
$$

Под $\tau_{0} \propto\left(T-T_{0}\right)$ теперь понимается отклонение температуры от ее критического значения (обозначения для двух моделей вводятся одинаково для удобства дальнейшего анализа), а коррелятор гауссова случайного шума $\zeta(t, \mathbf{x})$ выбирается в виде

$$
\left\langle\zeta(t, \mathbf{x}) \zeta\left(t^{\prime}, \mathbf{x}^{\prime}\right)\right\rangle=2 \lambda_{0} \delta\left(t-t^{\prime}\right) \delta^{(d)}\left(\mathbf{x}-\mathbf{x}^{\prime}\right) .
$$

Подобная модель может описывать некоторые универсальные аспекты фазового перехода первого рода в системе жидкость-пар [10], тогда как поведение системы вблизи критической точки определяется уравнением типа (3) с коррелятором (4) и нелинейностью $\varphi^{3}[1]$.

Различие в нормировке корреляторов шума в двух моделях объясняется соображениями удобства. Выбор коррелятора в форме (4) диктуется флуктуационно-диссипационной теоремой: одновременные корреляционные функции модели (3) описываются "статической" теорией $\varphi^{3}$ без дополнительных нормировочных множителей. Выбор коррелятора типа (2) обеспечивает равенство (с точностью до знака) констант связи при двух вершинах взаимодействия в теоретико-полевой формулировке модели (1) (см. выражение (10) ниже).

Взаимодействие с полем скорости вводится путем следующей замены:

$$
\partial_{t} \rightarrow \nabla_{t}=\partial_{t}+v_{i} \partial_{i}
$$

где $\nabla_{t}$ - лагранжева производная. В реальной ситуации поле скорости описывается нелинейным динамическим уравнением, например стохастическим уравнением Навье-Стокса со случайной силой, а анизотропия возникает из начальных и граничных условий.

В настоящей работе турбулентный поток мы моделируем случайным полем скорости, имеющим гауссово распределение с исчезающе малым временем корреляции и степенным спектром, а анизотропия вводится следующим образом [11]. Пусть $\mathbf{n}-$ постоянный единичный вектор, задающий выделенное направление (направление потока). Тогда любой вектор может быть разложен на две компоненты - перпендикулярную и параллельную потоку, например $\mathbf{x}=\mathbf{x}_{\perp}+\mathbf{n} x_{\|}$и $\mathbf{x}_{\perp} \cdot \mathbf{n}=0$. Поле скорости при этом выбирается в виде

$$
\mathbf{v}=\mathbf{n} v\left(t, \mathbf{x}_{\perp}\right),
$$

где $v\left(t, \mathbf{x}_{\perp}\right)$ - скалярная функция, не зависящая от $x_{\|}$. Легко заметить, что тогда условие поперечности (следствие несжимаемости жидкости) выполняется автоматически:

$$
\partial_{i} v_{i}=\partial_{\|} v\left(t, \mathbf{x}_{\perp}\right)=0 .
$$


Примем, что $v\left(t, \mathbf{x}_{\perp}\right)$ имеет гауссово распределение с нулевым средним и парным коррелятором

$$
\begin{aligned}
\left\langle v\left(t, \mathbf{x}_{\perp}\right) v\left(t^{\prime}, \mathbf{x}_{\perp}^{\prime}\right)\right\rangle & =\delta\left(t-t^{\prime}\right) \int \frac{d \mathbf{k}}{(2 \pi)^{d}} e^{i \mathbf{k} \cdot\left(\mathbf{x}-\mathbf{x}^{\prime}\right)} D_{v}(k)= \\
& =\delta\left(t-t^{\prime}\right) \int \frac{d \mathbf{k}_{\perp}}{(2 \pi)^{d-1}} e^{i \mathbf{k}_{\perp} \cdot\left(\mathbf{x}_{\perp}-\mathbf{x}_{\perp}^{\prime}\right)} \widetilde{D}_{v}\left(k_{\perp}\right), \quad k_{\perp}=\left|\mathbf{k}_{\perp}\right|,
\end{aligned}
$$

со скалярными функциями

$$
D_{v}(k)=2 \pi \delta\left(k_{\|}\right) \widetilde{D}_{v}\left(k_{\perp}\right), \quad \widetilde{D}_{v}\left(k_{\perp}\right)=D_{0} k_{\perp}^{-d+1-\xi} .
$$

Здесь $D_{0}>0$ - постоянная амплитуда, а $\xi$ - произвольный показатель, который вместе с $\varepsilon$ (отклонением размерности пространства от ее логарифмического значения) будет играть роль формального малого параметра РГ-разложения. ИК-регуляризация обеспечивается обрезанием нижней границы интегрирования по $k_{\perp}$ на значении $k_{\perp}=m$ (по размерности $\tau_{0} \propto m^{2}$ ). Конкретная форма ИК-регуляризации не играет особой роли; обрезание малых импульсов наиболее удобно для практических вычислений.

Естественная область определения для показателя $\xi-$ интервал $0<\xi<2$, для которого так называемая вихревая вязкость

$$
\mathcal{V}\left(\mathbf{r}_{\perp}\right)=\int \frac{d \mathbf{k}_{\perp}}{(2 \pi)^{d-1}}\left(1-e^{i \mathbf{k}_{\perp} \cdot \mathbf{r}_{\perp}}\right) \widetilde{D}_{v}\left(k_{\perp}\right)
$$

остается конечной при $m \rightarrow 0$. Этот интервал включает наиболее реалистичное значение $\xi=4 / 3$, отвечающее предположениям теории Колмогорова-Обухова, тогда как $\xi \rightarrow 2$ соответствует бэтчелоровскому пределу гладкой скорости. Добавим, что предложенный в работе [11] ансамбль (8), (9) является анизотропным аналогом известной модели Обухова-Крейчнана (см., например, [12]). В последнее время подобные модели с "синтетическими" ансамблями поля скорости приобрели большую популярность, так как с ними связан значительный прогресс в понимании явления развитой турбулентности, в особенности аномального скейлинга (см. обзоры [12], [13] и цитированную в них литературу).

Для обеспечения мультипликативной ренормируемости моделей необходимо "расщепить" лапласиан в (1) на параллельную и перпендикулярную составляющие, $\partial^{2} \rightarrow$ $\partial_{\perp}^{2}+f_{0} \partial_{\|}^{2}$, путем введения нового параметра $f_{0}>0$ (в случае анизотропной среды эти два члена будут ренормироваться по-разному). Такое расщепление нарушает $S O(d)$-симметрию наших уравнений; интерпретация этого факта может быть разной. Флуктуационные модели типа (1)-(4) являются феноменологическими и по построению должны включать все вклады, допустимые по размерности и симметрии, с независимыми коэффициентами. Поскольку исходная $S O(d)$-симметрия уравнений все равно редуцируется к $S O(d-1)$-симметрии в полных моделях с заменой (5), (6), естественно с самого начала рассматривать $S O(d-1)$-симметричные аналоги наших уравнений, что и сводится к введению произвольного $f_{0} \neq 1$. С другой стороны, можно было бы настаивать на изучении именно исходных моделей с $f_{0}=1$ и $S O(d)$-ковариантным (нерасщепленным) лапласианом. Тогда введение на данном этапе параметра $f_{0} \neq 1$ следует рассматривать лишь как технический трюк, 
гарантирующий мультипликативную ренормируемость модели и позволяющий вывести уравнения РГ. При этом сами эти уравнения должны решаться с физическим начальным условием $f_{0}=1$. Как мы увидим, ИК-устойчивая неподвижная точка уравнений РГ единственна для любого конкретного выбора параметров $\varepsilon$ и $\xi$, поэтому асимптотическое ИК-поведение для $f_{0}=1$ оказывается таким же, как и в общем случае с $f_{0} \neq 1$.

Согласно общей теореме (см., например, [1]) наши стохастические задачи (1), (2) и $(3),(4)$ после всех подстановок эквивалентны теоретико-полевым моделям с расширенным набором полей $\Phi=\left\{\varphi^{\prime}, \varphi, v\right\}$ и функционалами действия вида

$$
S_{1}(\Phi)=\varphi^{\prime}\left[-\partial_{t}-v \partial_{\|}+\lambda_{0}\left(\partial_{\perp}^{2}+f_{0} \partial_{\|}^{2}\right)-\lambda_{0} \tau_{0}\right] \varphi+\frac{\lambda_{0} g_{0} f_{0}^{1 / 4}}{2}\left[\left(\varphi^{\prime}\right)^{2} \varphi-\varphi^{2} \varphi^{\prime}\right]+S_{0}(v)
$$

$$
S_{2}(\Phi)=\lambda_{0}\left(\varphi^{\prime}\right)^{2}+\varphi^{\prime}\left[-\partial_{t}-v \partial_{\|}+\lambda_{0}\left(\partial_{\perp}^{2}+f_{0} \partial_{\|}^{2}\right)-\lambda_{0} \tau_{0}\right] \varphi-\frac{\lambda_{0} g_{0} f_{0}^{1 / 4}}{2} \varphi^{\prime} \varphi^{2}+S_{0}(v)
$$

соответственно, где мы выделили из зарядов $g_{0}$ множители $f_{0}^{1 / 4}$. В последних выражениях подразумеваются все необходимые интегрирования по $x=\{t, \mathbf{x}\}$ и суммирования по векторным индексам, например

$$
\varphi^{\prime} \partial_{\perp}^{2} \varphi=\int d t \int d \mathbf{x} \varphi^{\prime}(x) \partial_{\perp}^{2} \varphi(x)
$$

$\mathrm{K}$ действиям также добавлено дополнительное слагаемое $S_{0}(v)$, описывающее статистику поля скорости:

$$
\mathcal{S}_{0}(v)=-\frac{1}{2} \int d t \int d \mathbf{x}_{\perp} d \mathbf{x}_{\perp}^{\prime} v\left(t, \mathbf{x}_{\perp}\right) \widetilde{D}_{v}^{-1}\left(\left|\mathbf{x}_{\perp}-\mathbf{x}_{\perp}^{\prime}\right|\right) v\left(t, \mathbf{x}_{\perp}^{\prime}\right),
$$

где $\widetilde{D}_{v}^{-1}\left(r_{\perp}\right) \propto D_{0}^{-1} r_{\perp}^{2(1-d)-\xi}$.

Такая формулировка означает, что корреляционные функции исходных стохастических задач совпадают с функциями Грина теоретико-полевых моделей с функционалами действия (10) и (11), которые в свою очередь представляются функциональными средними с весом $e^{\mathcal{S}(\Phi)}$. Это позволяет нам применить к данным задачам теоретико-полевой метод РГ. Функции Грина с участием вспомогательного поля $\varphi^{\prime}$ отвечают функциям отклика в исходных стохастических задачах.

Модели (10) соответствует фейнмановская диаграммная техника с двумя типами затравочных пропагаторов (линий в диаграммах): пропагатор поля скорости $\langle v v\rangle_{0}$ был приведен ранее в (8), а функция отклика скалярного поля в частотно-импульсном представлении имеет вид

$$
\left\langle\varphi^{\prime} \varphi\right\rangle_{0}=\left\langle\varphi \varphi^{\prime}\right\rangle_{0}^{*}=\frac{1}{-i \omega+\lambda_{0}\left(k_{\perp}^{2}+f_{0} k_{\|}^{2}+\tau_{0}\right)}
$$

В импульсно-временном представлении она записывается как

$$
\left\langle\varphi^{\prime}\left(t^{\prime}\right) \varphi(t)\right\rangle_{0}=\theta\left(t-t^{\prime}\right) e^{-\lambda_{0}\left(k_{\perp}^{2}+f_{0} k_{\|}^{2}+\tau_{0}\right)\left(t-t^{\prime}\right)},
$$


где $\theta(\cdot)$ - функция Хевисайда (в данном выражении сразу видно, что функция отклика является запаздывающей).

Модель (10) также включает три типа вершин взаимодействия: $-\varphi^{\prime} \varphi^{2},\left(\varphi^{\prime}\right)^{2} \varphi$ и $-\varphi^{\prime}\left(v \partial_{\|}\right) \varphi$. Последняя в силу поперечности поля скорости равна $\varphi\left(v \partial_{\|}\right) \varphi^{\prime}$ (можно перенести производную на поле $\varphi^{\prime}$ путем интегрирования по частям).

Модели (11) отвечает диаграммная техника с тремя пропагаторами. Первые два совпадают с пропагаторами модели (10), кроме того, появляется дополнительный пропагатор

$$
\langle\varphi \varphi\rangle_{0}=\frac{2 \lambda_{0}}{\omega^{2}+\lambda_{0}^{2}\left(k_{\perp}^{2}+f_{0} k_{\|}^{2}+\tau_{0}\right)^{2}} .
$$

Вершин в данной модели только две: $-\varphi^{\prime} \varphi^{2}$ и прежняя вершина $-\varphi^{\prime}\left(v \partial_{\|}\right) \varphi$.

\section{3. КАНОНИЧЕСКИЕ РАЗМЕРНОСТИ}

Как известно, анализ УФ-расходимостей связан с анализом канонических размерностей (см., например, монографию [1]). Обычно в динамических задачах каноническая размерность любой величины $F$ полностью описывается двумя независимыми компонентами: частотной $d_{F}^{\omega}$ и импульсной $d_{F}^{k}$ размерностями. Однако в нашем случае из-за анизотропии необходимо ввести три независимые размерности $d_{F}^{\perp}, d_{F}^{\|}$ и $d_{F}^{\omega}$. Таким образом, для любой величины $F$ можно написать

$$
[F] \sim[T]^{-d_{F}^{\omega}}\left[L_{\perp}\right]^{-d_{F}^{\perp}}\left[L_{\|}\right]^{-d_{F}^{\|}}
$$

где $L_{\perp}$ и $L_{\|}$- независимые характерные масштабы в соответствующих подпространствах. Канонические размерности всех полей и констант, входящих в действие, можно найти, используя очевидные нормировочные соотношения $d_{k_{\perp}}^{\perp}=-d_{\mathbf{x}_{\perp}}^{\perp}=1$, $d_{k_{\perp}}^{\|}=-d_{\mathbf{x}_{\perp}}^{\|}=0, d_{k_{\perp}}^{\omega}=d_{k_{\|}}^{\omega}=0, d_{\omega}^{\omega}=-d_{t}^{\omega}=1$ и т. д., а также из условия безразмерности (для каждой из трех независимых размерностей в каноническом смысле) всех членов действия. Затем из этих трех независимых компонент можно составить суммарную размерность $d_{F}=d_{F}^{k}+2 d_{F}^{\omega}=d_{F}^{\perp}+d_{F}^{\|}+2 d_{F}^{\omega}$ (в свободной теории $\left.\partial_{t} \propto \partial^{2}\right)$, которая играет в ренормировке нашей модели ту же роль, что и обычная размерность $d_{F}^{k}$ в статике. Полный набор независимых канонических размерностей нужен, в частности, для нахождения полностью безразмерных параметров, от которых будут зависеть константы ренормировки. Канонические размерности (в том числе и для ренормированных параметров) для моделей (10) и (11) приведены в табл. 1 и табл. 2 соответственно.

В таблицах новая константа связи $w_{0}$ введена соотношением $D_{0}=w_{0} \lambda_{0} f_{0}$. Из табл. 1 видно, что модель (10) логарифмична (обе константы взаимодействия безразмерны) при $d=4, \xi=0$. Поэтому УФ-расходимости в корреляционных функциях проявляются как сингулярности при $\varepsilon \equiv 4-d \rightarrow 0, \xi \rightarrow 0$, например как полюсы по этим параметрам и их линейным комбинациям. Анализ табл. 2 показывает, что вторая модель логарифмична при $d=6, \xi=0$. Соответствующий параметр отклонения от логарифмичности мы также будем обозначать через $\varepsilon=6-d$.

Суммарная каноническая размерность произвольной 1-неприводимой функции Грина дается соотношением

$$
d_{\Gamma}=d+2-\sum_{\Phi} N_{\Phi} d_{\Phi}, \quad \sum_{\Phi} N_{\Phi} d_{\Phi}=N_{\varphi^{\prime}} d_{\varphi^{\prime}}+N_{\varphi} d_{\varphi}+N_{v} d_{v}
$$


ТАБлицА 1. Канонические размерности полей и параметров модели (10).

\begin{tabular}{c|c|c|c|c|c|c|c|c}
$F$ & $\varphi, \varphi^{\prime}$ & $v$ & $\lambda, \lambda_{0}$ & $f, f_{0}$ & $\tau, \tau_{0}$ & $g_{0}$ & $D_{0}$ & $w_{0}$ \\
\hline$d_{F}^{\omega}$ & 0 & 1 & 1 & 0 & 0 & 0 & 1 & 0 \\
\hline$d_{F}^{\perp}$ & $(d-1) / 2$ & 0 & -2 & 2 & 2 & $(4-d) / 2$ & $\xi$ & $\xi$ \\
\hline$d_{F}^{\|}$ & $1 / 2$ & -1 & 0 & -2 & 0 & 0 & -2 & 0 \\
\hline$d_{F}^{k}=d_{F}^{\perp}+d_{F}^{\|}$ & $d / 2$ & -1 & -2 & 0 & 2 & $(4-d) / 2$ & $-2+\xi$ & $\xi$ \\
\hline$d_{F}=2 d_{F}^{\omega}+d_{F}^{k}$ & $d / 2$ & 1 & 0 & 0 & 2 & $(4-d) / 2$ & $\xi$ & $\xi$
\end{tabular}

ТАБлицА 2. Канонические размерности полей и параметров модели (11).

\begin{tabular}{c|c|c|c|c|c|c|c|c}
$F$ & $\varphi$ & $\varphi^{\prime}$ & $v$ & $\lambda, \lambda_{0}$ & $f, f_{0}$ & $\tau, \tau_{0}$ & $g_{0}$ & $w_{0}$ \\
\hline$d_{F}^{\omega}$ & 0 & 0 & 1 & 1 & 0 & 0 & 0 & 0 \\
\hline$d_{F}^{\perp}$ & $(d-3) / 2$ & $(d+1) / 2$ & 0 & -2 & 2 & 2 & $(6-d) / 2$ & $\xi$ \\
\hline$d_{F}^{\|}$ & $1 / 2$ & $1 / 2$ & -1 & 0 & -2 & 0 & 0 & 0 \\
\hline$d_{F}^{k}=d_{F}^{\perp}+d_{F}^{\|}$ & $d / 2-1$ & $d / 2+1$ & -1 & -2 & 0 & 2 & $(6-d) / 2$ & $\xi$ \\
\hline$d_{F}=2 d_{F}^{\omega}+d_{F}^{k}$ & $d / 2-1$ & $d / 2+1$ & 1 & 0 & 0 & 2 & $(6-d) / 2$ & $\xi$
\end{tabular}

Здесь $N_{\Phi}=\left\{N_{\varphi}, N_{\varphi^{\prime}}, N_{v}\right\}$ - чи́сла соответствующих полей, входящих в функцию Г. Суммарная размерность $d_{\Gamma}$ в логарифмической теории (т. е. при $\varepsilon=\xi=0$ ) является формальным индексом УФ-расходимости, $\delta_{\Gamma}=\left.d_{\Gamma}\right|_{\varepsilon=\xi=0}$. Поверхностные расходимости, для устранения которых требуется введение контрчленов, могут возникать только в тех диаграммах, для которых индекс расходимости - неотрицательное целое число.

Соображения размерности могут быть дополнены некоторыми наблюдениями, позволяющими сократить количество реально необходимых контрчленов. Например, можно заметить, что для модели с взаимодействием Грибова (10) все 1-неприводимые диаграммы отличны от нуля, только если они содержат оба поля $\varphi$ и $\varphi^{\prime}$, т. е. одновременно $N_{\varphi} \geqslant 1$ и $N_{\varphi^{\prime}} \geqslant 1$. В противном случае диаграммы обязательно содержат замкнутый цикл запаздывающих пропагаторов $\left\langle\varphi \varphi^{\prime}\right\rangle_{0}$ и, следовательно, исчезают, не требуя контрчленов. Подобное замечание можно сделать и для модели (11): здесь также все 1-неприводимые функции без поля $\varphi^{\prime}$ исчезают и не требуют контрчленов. В частности, для обеих моделей любые функции, содержащие только поле скорости, подпадают под эти правила, и поэтому поле остается гауссовым. Функцию $\left\langle\varphi \varphi^{\prime} v v\right\rangle$ тоже можно не рассматривать, так как соответствующий контрчлен $\varphi \varphi^{\prime} v^{2}$ запрещен галилеевой инвариантностью. Аналогичная ситуация имеет место и для функций $\left\langle\varphi^{\prime} v\right\rangle$ и $\left\langle\varphi^{\prime} v v\right\rangle$ в модели (11). Для первой функции контрчлен обязательно содержит пространственную производную, сворачивающуюся по индексу с полем скорости; это дает ноль в силу условия поперечности (7). Для второй из этих функций единственный контрчлен, не запрещенный галиле- 
евой инвариантностью и поперечностью, имеет вид $\varphi^{\prime}\left(\partial_{i} v_{k}\right)\left(\partial_{k} v_{i}\right)$. В общем случае он нетривиален, но для нашего поля скорости вида (6) он также обращается в ноль в силу $(7):\left(\partial_{i} n_{k} v\right)\left(\partial_{k} n_{i} v\right)=\left(n_{i} \partial_{i} v\right)\left(n_{k} \partial_{k} v\right)=0$. Кроме того, контрчлены типа $\varphi \partial_{t} \varphi^{\prime}$ и $\varphi\left(v \partial_{\|}\right) \varphi^{\prime}$ могут появляться в ренормированном действии только в виде галилеево-инвариантной комбинации типа $\varphi \nabla_{t} \varphi^{\prime}$.

Выясним, в каких 1-неприводимых функциях могут содержаться УФ-расходимости, начав с модели (10). Формула (15) примет вид $\delta_{\Gamma}=6-2 N_{\varphi}-2 N_{\varphi^{\prime}}-N_{v}$. Выпишем все возможные функции (далее приведены сами функции, индексы расходимости и возможные типы контрчленов):

$$
\begin{array}{lll}
\left\langle\varphi^{\prime} \varphi\right\rangle, & \delta=2, & \varphi^{\prime} \partial_{t} \varphi, \varphi^{\prime} \partial^{2} \varphi, \varphi^{\prime} \varphi, \\
\left\langle\varphi^{\prime} \varphi \varphi\right\rangle, & \delta=0, & \varphi^{\prime} \varphi^{2}, \\
\left\langle\varphi^{\prime} \varphi^{\prime} \varphi\right\rangle, & \delta=0, & \left(\varphi^{\prime}\right)^{2} \varphi, \\
\left\langle\varphi^{\prime} \varphi v\right\rangle, & \delta=1, &
\end{array}
$$

для функции $\left\langle\varphi^{\prime} \varphi v\right\rangle$ контрчлен с необходимостью приводится к форме $\varphi^{\prime}\left(v_{i} \partial_{i}\right) \varphi=$ $\varphi^{\prime}\left(v \partial_{\|}\right) \varphi$. Все такие члены представлены в действии $(10)$, поэтому оказывается, что наша первая модель является мультипликативно-ренормируемой.

Обратимся ко второй модели. Формула (15) для модели (11) принимает вид $\delta_{\Gamma}=8-2 N_{\varphi}-4 N_{\varphi}^{\prime}-N_{v}$. Как уже указывалось выше, некоторые допустимые по формальному индексу расходимости в действительности запрещены галилеевой инвариантностью. В итоге остаются следующие 1-неприводимые функции (вновь приведены сами функции, индексы расходимости и возможные типы контрчленов):

$$
\begin{aligned}
& \left\langle\varphi^{\prime} \varphi\right\rangle, \quad \delta=2, \quad \varphi^{\prime} \varphi, \varphi^{\prime} \partial_{\perp}^{2} \varphi, \varphi^{\prime} \partial_{\|}^{2} \varphi, \\
& \left\langle\varphi^{\prime} \varphi^{\prime}\right\rangle, \quad \delta=0, \quad\left(\varphi^{\prime}\right)^{2}, \\
& \left\langle\varphi^{\prime} \varphi \varphi\right\rangle, \quad \delta=0, \quad \varphi^{\prime} \varphi^{2}, \\
& \left\langle\varphi^{\prime} \varphi v\right\rangle, \quad \delta=1, \quad \varphi^{\prime}\left(v \partial_{\|}\right) \varphi, \\
& \left\langle\varphi^{\prime}\right\rangle, \quad \delta=4, \quad \varphi^{\prime} \text {. }
\end{aligned}
$$

В действии (11) представлены все такие члены, кроме последнего, поэтому для обеспечения мультипликативности ренормировки к нему следовало бы добавить линейное по полю $\varphi^{\prime}$ слагаемое, т. е. ввести его взаимодействие с некоторым внешним полем $h$ (важно, что введение такого вклада не порождает новых расходимостей, т. е. не отражается на уже вычисленных при $h=0$ контрчленах). Это приведет к некоторым неоднородным вкладам в уравнениях РГ, не влияющим на вид $\beta$-функций и, в конечном счете, на вид критических размерностей. Поэтому в дальнейшем мы будем просто игнорировать этот вклад, считая (с приведенной оговоркой) модель (11) мультипликативно-ренормируемой, как и модель (10).

В итоге ренормированные действия наших двух моделей можно записать в виде

$$
\begin{aligned}
& S_{1 \mathrm{R}}(\Phi)=\varphi^{\prime} {\left[-Z_{1} \partial_{t}-Z_{6} v \partial_{\|}+\lambda\left(Z_{2} \partial_{\perp}^{2}+Z_{3} f \partial_{\|}^{2}-Z_{4} \tau\right)\right] \varphi+} \\
&+Z_{5} \frac{\lambda g f^{1 / 4} \mu^{\varepsilon / 2}}{2}\left[\left(\varphi^{\prime}\right)^{2} \varphi-\varphi^{2} \varphi^{\prime}\right]+S_{\mathrm{R}}(v), \\
& S_{2 \mathrm{R}}(\Phi)=Z_{7} \lambda\left(\varphi^{\prime}\right)^{2}+\varphi^{\prime}\left[-Z_{1} \partial_{t}-Z_{6} v \partial_{\|}+\lambda\left(Z_{2} \partial_{\perp}^{2}+Z_{3} f \partial_{\|}^{2}-Z_{4} \tau\right)\right] \varphi- \\
&-Z_{5} \frac{\lambda g f^{1 / 4} \mu^{\varepsilon / 2}}{2} \varphi^{\prime} \varphi^{2}+S_{\mathrm{R}}(v) .
\end{aligned}
$$


Здесь $S_{\mathrm{R}}(v)$ - действие $S_{0}(v)$, выраженное в ренормированных переменных; эта часть действия не ренормируется, потому что, как отмечалось выше, в наших моделях нет контрчленов, построенных только из поля скорости. Величины $\lambda, \tau, f, w$ и $g$ - ренормированные аналоги исходных неренормированных параметров (отмеченных индексом 0). Напомним, галилеева инвариантность требует, чтобы выражение $\partial_{t}+v \partial_{\|}$входило в контрчлен как одно целое. Отсюда вытекает тождество $Z_{1}=Z_{6}$, которое будет подтверждено прямым вычислением в однопетлевом приближении; пока же для общности мы будем различать эти константы.

Выражения (16) и (17) эквивалентны мультипликативной ренормировке полей $\varphi \rightarrow \varphi Z_{\varphi}, \varphi^{\prime} \rightarrow \varphi^{\prime} Z_{\varphi}^{\prime}, v \rightarrow v Z_{v}$ и параметров

$$
\lambda_{0}=\lambda Z_{\lambda}, \quad \tau_{0}=\tau Z_{\tau}, \quad f_{0}=f Z_{u}, \quad g_{0}=g \mu^{\varepsilon / 2} Z_{g}, \quad w_{0}=w \mu^{\xi} Z_{w},
$$

где $\mu$ - добавочный произвольный параметр ренормированной теории (это свободный параметр, размерность его совпадает с размерностью импульса; будем считать, что $\left.d_{\mu}^{\perp}=1, d_{\mu}^{\|}=0\right)$. Константы $Z$ в модели (16) и в формулах (18) соотносятся следующим образом:

$$
\begin{aligned}
& Z_{1}=Z_{\varphi^{\prime}} Z_{\varphi}, \quad Z_{2}=Z_{\varphi^{\prime}} Z_{\lambda} Z_{\varphi}, \quad Z_{3}=Z_{\varphi^{\prime}} Z_{\lambda} Z_{f} Z_{\varphi}, \\
& Z_{4}=Z_{\varphi^{\prime}} Z_{\lambda} Z_{\tau} Z_{\varphi}, \quad Z_{5}=Z_{\lambda} Z_{g} Z_{f}^{1 / 4} Z_{\varphi^{\prime}} Z_{\varphi}^{2}, \quad Z_{6}=Z_{\varphi^{\prime}} Z_{v} Z_{\varphi}, \\
& 1=Z_{v}^{2} Z_{w}^{-1} Z_{f}^{-1} Z_{\lambda}^{-1} \text {. }
\end{aligned}
$$

Последнее равенство - следствие отсутствия ренормировки вклада $S_{0}(v)=S_{\mathrm{R}}(v)$.

Для модели (17) соотношения (19) остаются верными, но к ним добавляется выражение для константы ренормировки $Z_{7}$ :

$$
Z_{7}=Z_{\lambda}\left(Z_{\varphi^{\prime}}\right)^{2}
$$

Кроме того, для первой модели в силу симметрии потенциала присутствует условие связи констант ренормировки полей $\varphi^{\prime}$ и $\varphi$ :

$$
Z_{\varphi^{\prime}}=Z_{\varphi}
$$

Наконец, из галилеевой инвариантности вытекает равенство $Z_{1}=Z_{6}$ (оно будет проверено прямым вычислением в первом порядке), из которого в свою очередь следует отсутствие ренормировки поля скорости: $Z_{v}=1$.

Введение констант ренормировки устраняет все сингулярности по $\varepsilon, \xi$ так, что корреляционные функции ренормированной модели имеют конечный предел при $\varepsilon, \xi=0$. Для доопределения конечной части при вычислении констант ренормировки мы будем пользоваться схемой минимальных вычитаний (MB), в которой они имеют вид $Z_{i}=1+$ только части, имеющие полюсы по $\varepsilon, \xi$. Коэффициенты при этих полюсах зависят только от безразмерных ренормированных констант взаимодействия $g$ и $w$.

\section{4. ВЫЧИСЛЕНИЕ КОНСТАНТ РЕНОРМИРОВКИ}

Обратимся к вычислению констант ренормировки $Z$ в однопетлевом приближении. Поясним используемые ниже диаграммные обозначения. Волнистой линией обозначен пропагатор $\langle v v\rangle_{0}$, прямой линией со штрихом - пропагатор $\left\langle\varphi^{\prime} \varphi\right\rangle_{0}$ (причем штрих относится к полю $\varphi^{\prime}$ ), а прямой линии без штрихов отвечает пропагатор $\langle\varphi \varphi\rangle_{0}$, присутствующий лишь во второй модели. Все диаграммные элементы 
должны быть выражены через ренормированные переменные (16)-(20). Однако в однопетлевом приближении константы $Z$ во всех затравочных слагаемых нужно брать в первом порядке по $g^{2}$ и $w$ (реально разложение идет не по $g$, а по $g^{2}$ ), а в диаграммах их просто нужно заменить на единицы. Таким образом, переход к ренормированным переменным в диаграммах сводится к заменам $\lambda_{0} \rightarrow \lambda, f_{0} \rightarrow f$, $\tau_{0} \rightarrow \tau, g_{0} \rightarrow g \mu^{\varepsilon / 2}$ и $w_{0} \rightarrow w \mu^{\xi}$.

Обратимся к модели (16). Приведем все 1-неприводимые функции, необходимые для расчета полного набора констант ренормировки, с однопетлевой точностью. Уравнение Дайсона для 1-неприводимой функции Грина $\left\langle\varphi^{\prime} \varphi\right\rangle_{1-i r}$ в таком приближении примет вид

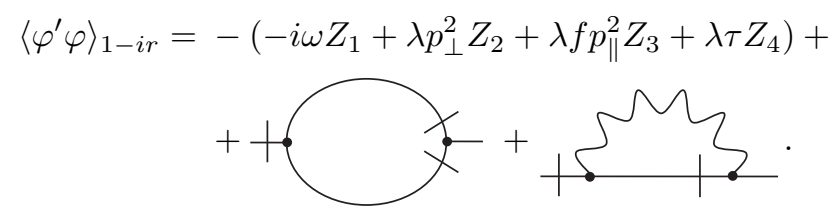

Для 1-неприводимой корреляционной функции $\left\langle\varphi^{\prime} \varphi^{\prime} \varphi\right\rangle_{1-i r}$ имеем

$$
\left\langle\varphi^{\prime} \varphi^{\prime} \varphi\right\rangle_{1-i r}=g \lambda f^{1 / 4} Z_{5}+2{ }_{x}^{x}
$$

Наконец, 1-неприводимая функция $\left\langle\varphi^{\prime} v \varphi\right\rangle_{1-i r}$, нужная лишь для того чтобы выполнить обещанную выше проверку следствий из галилеевой инвариантности, такова:

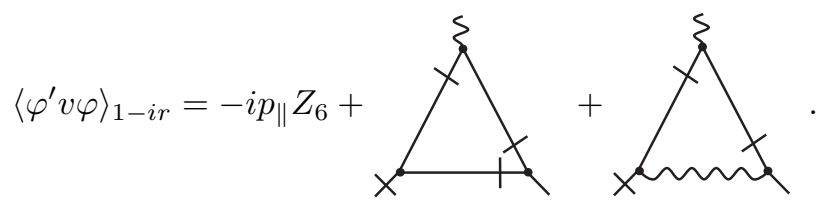

Мы не будем подробно описывать процесс вычисления этих диаграмм, лишь прокомментируем основные моменты. Сразу можно заметить, что вторая и третья диаграммы в (23) и вторая диаграмма в (24) оказываются УФ-конечными, следовательно, они не вносят вклад в константы ренормировки $Z$. Действительно, благодаря свойству (7) поперечности поля скорости производная $\partial_{\|}$в тройной вершине $-\varphi^{\prime}\left(v \partial_{\|}\right) \varphi$ может быть переброшена на внешнюю линию за счет интегрирования по частям. Таким образом, любая диаграмма, содержащая $n$ внешних тройных вершин $-\varphi^{\prime}\left(v \partial_{\|}\right) \varphi$, будет содержать множитель $p_{\|}^{n}$ с внешним импульсом $p_{\|}$. Это понижает размерность интеграла, и он может стать УФ-конечным, что и происходит с указанными диаграммами (для них $n=2$ ).

Более того, так как пропагатор (13) запаздывающий, а коррелятор (8) содержит $\delta$-функцию по времени, вторые диаграммы в (23) и (24) содержат замкнутые циклы функций Хевисайда по времени, а значит, тождественно обращаются в ноль. Такой аргумент, однако, неприменим ко второй диаграмме в (22), требующей более тщательного рассмотрения. Аналитическое выражение для этой диаграммы имеет 
вид

$$
I_{1}=-\lambda f w \mu^{\xi} \int \frac{d \mathbf{k}_{\perp} d k_{\|} d \eta}{(2 \pi)^{d}} \frac{\delta\left(k_{\|}\right) p_{\|}\left(p_{\|}-k_{\|}\right)}{-i \eta+\lambda\left[\left(p_{\perp}-k_{\perp}\right)^{2}+f\left(p_{\|}-k_{\|}\right)^{2}+\tau\right]} \cdot \frac{1}{k_{\perp}^{d-1+\xi}} .
$$

Заметим что вклад этой диаграммы в $Z_{1}, Z_{2}$ и $Z_{4}$ равен нулю. От внешней частоты она явно не зависит (внешнюю частоту можно "пропустить" по пропагатору скорости, а там нет зависимости от частоты), а независимость от $\tau$ и $p_{\perp}^{2}$ станет очевидной после интегрирования по внутренней частоте $\eta$. Покажем это, воспользовавшись равенством

$$
\frac{1}{2 \pi} \int \frac{d \eta}{-i \eta+A}=\frac{1}{2}
$$

которое фактически является доопределением $\theta(0)=1 / 2$; его можно оправдать симметричностью коррелятора $\langle v v\rangle_{0}$. После применения этого равенства приходим к выражению

$$
I_{1}=-\frac{\lambda f w \mu^{\xi} p_{\|}^{2}}{2(2 \pi)^{d-1}} \int_{k_{\perp} \geqslant m} \frac{d \mathbf{k}_{\perp}}{k_{\perp}^{d-1+\xi}}
$$

которое легко интегрируется в сферических координатах:

$$
I_{1}=-\frac{p_{\|}^{2}}{2 \xi} \frac{f \lambda w}{(2 \pi)^{d-1}} S_{d-1}\left(\frac{\mu}{m}\right)^{\xi} \simeq-p_{\|}^{2} \frac{\lambda f w}{\xi},
$$

где $S_{d}=2 \pi^{d / 2} / \Gamma(d / 2)$ - площадь единичной сферы в $d$-мерном пространстве. В формуле для $I_{1}$ мы перешли к более удобной константе связи: $w \rightarrow$ $w S_{d-1} / 2(2 \pi)^{d-1} \rightarrow w / 4 \pi^{2}$. При этом в соответствии с правилами схемы МВ мы заменили $d$ его логарифмическим значением $d=4$, отбросив тем самым множитель порядка $1+O(\varepsilon)$, который не содержит полюс по $\varepsilon$ и в нашем приближении не отражается на виде констант ренормировки. Теперь нетрудно найти, что вклад этой диаграммы в $Z_{3}$ равен $-w / \xi$.

Рассмотрим теперь первую диаграмму в (22). Аналитическое выражение для нее имеет вид

$$
\begin{aligned}
I_{2}= & -\left(\lambda g f^{1 / 4} \mu^{\varepsilon / 2}\right)^{2} \int \frac{d \mathbf{k}_{\perp} d k_{\|} d \eta}{(2 \pi)^{d+1}} \frac{1}{-i(\omega+\eta)+\lambda\left[\left(p_{\perp} / 2+k_{\perp}\right)^{2}+f\left(p_{\|} / 2+k_{\|}\right)^{2}+\tau\right]} \times \\
& \times \frac{1}{i \eta+\lambda\left[\left(p_{\perp} / 2-k_{\perp}\right)^{2}+f\left(p_{\|} / 2-k_{\|}\right)^{2}+\tau\right]} .
\end{aligned}
$$

Выполнив интегрирование по внутренней частоте $\eta$ путем взятия вычетов и сделав стандартную для этих моделей замену $q_{\perp}=k_{\perp}, q_{\|}=f^{1 / 2} k_{\|}$, после интегрирования по $q$ получаем $(d=4-\varepsilon)$

$$
\begin{aligned}
I_{2} & =-\left(\lambda g f^{1 / 4} \mu^{\varepsilon / 2}\right)^{2} \frac{1}{(2 \pi)^{d}} \frac{1}{2 \lambda f^{1 / 2}}\left(-\frac{i \omega}{2 \lambda}+\tau+\frac{p_{\perp}^{2}}{4}+\frac{f p_{\|}^{2}}{4}\right)^{1-\varepsilon / 2} \pi^{d / 2} \Gamma\left(-1+\frac{\varepsilon}{2}\right)= \\
& =-\frac{u \lambda}{2}\left(-\frac{i \omega}{2 \lambda}+\tau+\frac{p_{\perp}^{2}}{4}+\frac{f p_{\|}^{2}}{4}\right)^{1-\varepsilon / 2}(2 \mu)^{\varepsilon} \pi^{\varepsilon / 2} \Gamma\left(-1+\frac{\varepsilon}{2}\right),
\end{aligned}
$$

где также введен более удобный заряд $u=g^{2} S_{d} / 2(2 \pi)^{d} \rightarrow g^{2} / 16 \pi^{2}$ (как и в случае $w$, значение $d$ заменено его логарифмическим значением в согласии со схемой МВ). 
Отсюда находим вклады этой диаграммы в $Z_{1}, Z_{2}, Z_{3}$ и $Z_{4}$, paвные $u / 4 \varepsilon, u / 8 \varepsilon, u / 8 \varepsilon$ и $u / 2 \varepsilon$ соответственно.

Рассмотрим единственную оставшуюся диаграмму в (23). Ее индекс расходимости равен нулю, поэтому можно сделать все внешние импульсы и частоты равными нулю, получив аналитическое выражение

$$
I_{3}=-\left(\lambda g f^{1 / 4} \mu^{\varepsilon / 2}\right)^{3} \int \frac{d \mathbf{k}_{\perp} d k_{\|} d \eta}{(2 \pi)^{d+1}} \frac{1}{\left[-i \eta+\lambda\left(k_{\perp}^{2}+f k_{\|}^{2}+\tau\right)\right]^{2}} \frac{1}{i \eta+\lambda\left(k_{\perp}^{2}+f k_{\|}^{2}+\tau\right)} .
$$

После интегрирования по $\eta$ оно принимает вид

$$
I_{3}=-\frac{\lambda g^{3} f^{1 / 4} \mu^{3 \varepsilon / 2}}{4(2 \pi)^{d}} \int \frac{d \mathbf{q}}{q^{2}+\tau} .
$$

Наконец, выполнив интегрирование по $q$, приходим к соотношению

$$
I_{3} \simeq-\frac{\lambda u f^{1 / 4}}{2 \varepsilon}
$$

таким образом, вклад этой диаграммы в $Z_{5}$ составляет $u / \varepsilon$ с учетом коэффициента 2 в $(23)$.

Обратимся к диаграмме в (24). Так как нас интересует только ее зависимость от продольных импульсов, все прочие внешние параметры можно положить равными нулю. У этой диаграммы три внешних "хвоста", т. е. число независимых внешних импульсов в общем случае равно двум. Однако, чтобы найти константу ренормировки, достаточно рассматривать только один импульс, текущий между “хвостами” $\varphi$ и $\varphi$ '. Действительно, пусть импульс $p_{\|}$течет от $\varphi^{\prime} \mathrm{\kappa} \varphi$, а импульс $q_{\|}-$от $\varphi^{\prime} \mathrm{k} v$. Тогда выражение для расходящейся части диаграммы будет иметь вид $\alpha p_{\|}+\beta q_{\|}$, где $\alpha$ и $\beta$ - искомые величины. Однако контрчлен при $\beta$ в выражении $\alpha \varphi^{\prime}\left(v \partial_{\|}\right) \varphi+\beta \varphi^{\prime}\left(\partial_{\|} v\right) \varphi$ оказывается тождественно равным нулю, поскольку $\partial_{\|} v=0$ согласно (7). Выпишем аналитическое выражение для этой диаграммы:

$$
I_{4}=\left(\lambda g f^{1 / 4} \mu^{\varepsilon / 2}\right)^{2} \int \frac{d \mathbf{k}_{\perp} d k_{\|} d \eta}{(2 \pi)^{d+1}} \frac{-i k_{\|}}{\left[-i \eta+\lambda\left(k_{\perp}^{2}+f k_{\|}^{2}\right)\right]^{2}} \frac{1}{i \eta+\lambda\left(k_{\perp}^{2}+f k_{\|}^{2}\right)} .
$$

После интегрирования по $\eta$ и $\mathbf{k}_{\perp}$ и упоминавшейся выше замены приходим к выражению

$$
I_{4}=-\frac{i}{(2 \pi)^{d}} \frac{f^{-1 / 2} g^{2} \mu^{\varepsilon}}{4} \int d q_{\|} q_{\|}\left(\left(q_{\|}-\frac{f^{1 / 2} p_{\|}}{2}\right)^{2}+\frac{f p_{\|}}{2}\right)^{(d-5) / 2} \pi^{(d-1) / 2} \Gamma\left(\frac{5-d}{2}\right) .
$$

Вычитая и добавляя к $q_{\|}$в подынтегральном выражении $f^{1 / 2} p_{\|} / 2$, разбиваем интеграл на два. Первый равен нулю по соображениям четности, а второй легко вычислить:

$$
I_{4}=\frac{-i p_{\|}}{(2 \pi)^{d}} \frac{g^{2} \mu^{\varepsilon}}{8} \pi^{d / 2} \Gamma\left(\frac{d-4}{2}\right)=\frac{-i p_{\|}}{8} u \pi^{\varepsilon / 2}(2 \mu)^{\varepsilon} \Gamma\left(\frac{d-4}{2}\right),
$$

где мы также перешли к введенной ранее константе связи $u=(g / 4 \pi)^{2}$. Отсюда получаем $Z_{6}=1+u / 4 \varepsilon$. 
Приведем однопетлевые результаты для всех $Z$ :

$$
\begin{aligned}
Z_{1} & =1+\frac{u}{4 \varepsilon}, & Z_{2} & =1+\frac{u}{8 \varepsilon}, \\
Z_{4} & =1+\frac{u}{2 \varepsilon}, & Z_{3} & =1+\frac{u}{8 \varepsilon}-\frac{w}{\xi}, \\
Z_{5} & =1+\frac{u}{\varepsilon}, & Z_{6} & =1+\frac{u}{4 \varepsilon} .
\end{aligned}
$$

Таким образом, для первой модели в однопетлевом приближении мы прямым расчетом подтвердили точное равенство $Z_{1}=Z_{6}$, другими словами, $Z_{v}=1$ (см. формулы (19)).

Обратимся теперь к модели (17) и приведем все необходимые для расчета ее констант ренормировки 1-неприводимые функции в однопетлевом приближении. Уравнение Дайсона для $\left\langle\varphi^{\prime} \varphi\right\rangle_{1-i r}$ здесь имеет вид

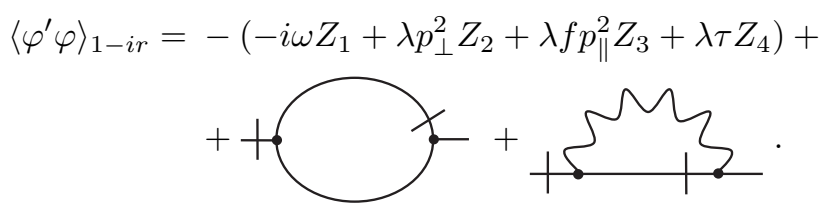

Для 1-неприводимой корреляционной функции $\left\langle\varphi^{\prime} \varphi \varphi\right\rangle_{1-i r}$ имеем

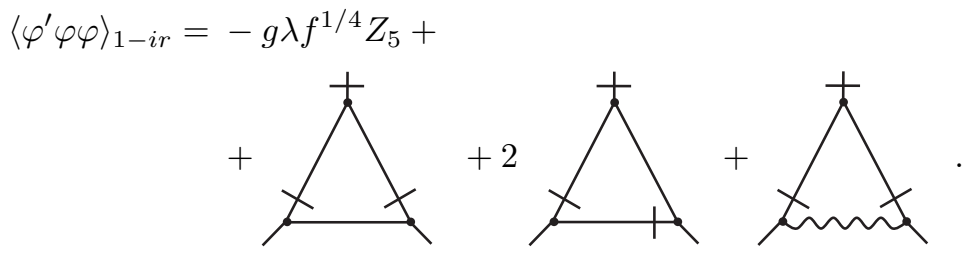

Функция, необходимая для проверки следствий галилеевой инвариантности, такова:

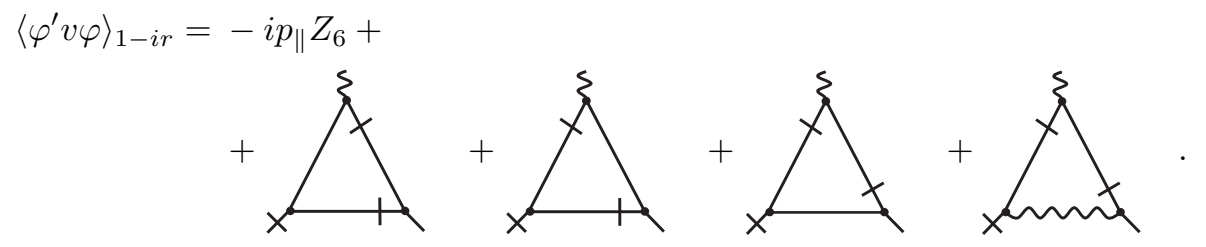

Наконец, для 1-неприводимой функции Грина $\langle\varphi \varphi\rangle_{1-i r}$ имеем

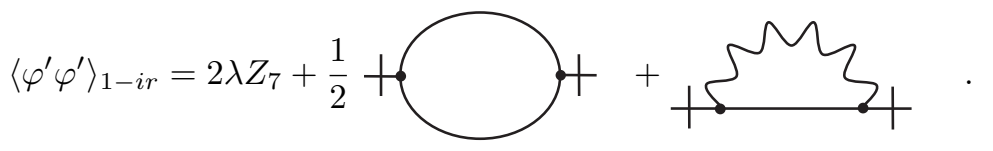

Здесь благодаря специфическим особенностям нашей системы некоторые диаграммы также исчезают или оказываются УФ-конечными и, следовательно, не дают вклада в ренормировочные константы. Например, за счет наличия двух внешних тройных вершин $-\varphi^{\prime}\left(v \partial_{\|}\right) \varphi$ последние диаграммы в (27)-(29) УФ-конечны.

Сделаем некоторые замечания по поводу вычисления оставшихся диаграмм. Рассмотрим ренормировку 1-неприводимой функции $\left\langle\varphi^{\prime} \varphi\right\rangle_{1-i r}$. Вторые диаграммы 
в (22) и (26) совпадают, так что мы просто используем уже полученный ранее результат. Новые заряды введем следующим образом:

$$
u=g^{2} \frac{S_{d}}{2(2 \pi)^{d}} \rightarrow \frac{g^{2}}{128 \pi^{3}}, \quad w \rightarrow w \frac{S_{d-1}}{2(2 \pi)^{d-1}} \rightarrow \frac{w}{24 \pi^{3}},
$$

поскольку теперь $d$ нужно заменить логарифмическим значением $d=6$. Аналитическое выражение для первой диаграммы следующее:

$$
\begin{aligned}
I_{5}=( & \left.-\lambda g f^{1 / 4} \mu^{\varepsilon / 2}\right)^{2} \int \frac{d \mathbf{k}_{\perp} d k_{\|} d \eta}{(2 \pi)^{d+1}} \frac{1}{-i(\omega+\eta)+\lambda\left[\left(p_{\perp} / 2+k_{\perp}\right)^{2}+f\left(p_{\|} / 2+k_{\|}\right)^{2}+\tau\right]} \times \\
& \times \frac{2 \lambda}{\eta^{2}+\lambda^{2}\left[\left(p_{\perp} / 2-k_{\perp}\right)^{2}+f\left(p_{\|} / 2-k_{\|}\right)^{2}+\tau\right]^{2}} .
\end{aligned}
$$

После интегрирования по внутренней частоте $\eta$ и перехода к переменной $q$ получаем

$$
\begin{aligned}
I_{5}= & \frac{\lambda g^{2} \mu^{\varepsilon}}{2(2 \pi)^{d}} \int d \mathbf{q}_{\perp} d q_{\|} \frac{1}{\left(p_{\perp} / 2-q_{\perp}\right)^{2}+\left(f^{1 / 2} p_{\|} / 2-q_{\|}\right)^{2}+\tau} \times \\
& \times \frac{1}{\omega / 2 i \lambda+\left[q_{\perp}^{2}+q_{\|}^{2}+\tau+\left(p_{\perp}^{2}+f p_{\|}^{2}\right) / 4\right]} .
\end{aligned}
$$

Вводя обозначения $\tilde{p}_{\perp}=p_{\perp}, \tilde{p}_{\|}=f^{1 / 2} p_{\|}$и применяя формулу Фейнмана

$$
\frac{1}{A^{\alpha} B^{\beta}}=\frac{\Gamma(\alpha+\beta)}{\Gamma(\alpha) \Gamma(\beta)} \int_{0}^{1} d t \frac{t^{\alpha-1}(1-t)^{\beta-1}}{[t A+(1-t) B]^{\alpha+\beta}},
$$

получаем

$$
I_{5}=\frac{\lambda g^{2} \mu^{\varepsilon}}{2(2 \pi)^{d}} \int d \mathbf{q} \int_{0}^{1} \frac{d t}{\left[k^{2}-t(\tilde{p} q)+\tau+\tilde{p}^{2} / 4+(\omega / 2 i \lambda)(1-t)\right]^{2}} .
$$

Интеграл с квадратичной формой по импульсу в знаменателе вычисляется стандартным образом, а после выделения вклада, содержащего только полюс, легко выполняется и оставшееся интегрирование по $t$. Окончательно получаем

$$
I_{5} \simeq-\frac{\lambda g^{2}}{(2 \pi)^{6}} \frac{\pi^{3}}{\varepsilon}\left(\tau+\frac{\omega}{4 i \lambda}+\frac{p_{\perp}^{2}+f p_{\|}^{2}}{6}\right) \simeq-\frac{\lambda u}{\varepsilon}\left(2 \tau-\frac{i \omega}{2 \lambda}+\frac{p_{\perp}^{2}+f p_{\|}^{2}}{3}\right) .
$$

Теперь нетрудно найти вклады этой диаграммы в $Z_{1}, Z_{2}, Z_{3}$ и $Z_{4}$ : они равны $-u / 2 \varepsilon$, $-u / 3 \varepsilon,-u / 3 \varepsilon$ и $-2 u / \varepsilon$ соответственно.

Первая и вторая диаграммы в (27) вычисляются практически одинаково, так что остановимся только на первой из них. Индекс расходимости для этих диаграмм равен нулю, поэтому мы можем положить равными нулю внешние частоту и импульс ( $\tau$ оставляем для ИК-регуляризации). Тогда получаем следующее аналитическое выражение для первой диаграммы:

$$
I_{6}=\left(-\lambda g f^{1 / 4} \mu^{\varepsilon / 2}\right)^{3} \int \frac{d \mathbf{k}_{\perp} d k_{\|} d \eta}{(2 \pi)^{d+1}} \frac{2 \lambda}{\eta^{2}+\lambda^{2}\left[\left(p_{\perp} / 2-k_{\perp}\right)^{2}+f k_{\|}^{2}+\tau\right]^{2}} \times
$$




$$
\begin{gathered}
\times \frac{1}{-i \eta+\lambda\left(k_{\perp}^{2}+f k_{\|}^{2}+\tau\right)} \frac{1}{i \eta+\lambda\left(k_{\perp}^{2}+f k_{\|}^{2}+\tau\right)}= \\
=-\frac{2 \lambda^{4} g^{3} f^{1 / 4} \mu^{3 \varepsilon / 2}}{(2 \pi)^{d+1}} \int \frac{d \mathbf{q} d \eta}{\left[\eta^{2}+\lambda^{2}\left(q^{2}+\tau\right)\right]^{2}} .
\end{gathered}
$$

Проинтегрировав по $\eta$, получаем выражение, которое нетрудно проинтегрировать и по импульсу:

$$
I_{6}=-\frac{g^{3} f^{1 / 4} \lambda \mu^{3 \varepsilon / 2} \pi}{(2 \pi)^{d+1}} \int \frac{d \mathbf{q}}{\left(q^{2}+\tau\right)^{3}}=-\frac{g^{3} f^{1 / 4} \lambda \mu^{3 \varepsilon / 2} \pi}{2(2 \pi)^{d+1}} \tau^{-\varepsilon / 2} \Gamma\left(\frac{\varepsilon}{2}\right) \pi^{d / 2} \simeq-\frac{g f^{1 / 4} \lambda u}{\varepsilon} .
$$

Таким образом, вклад диаграммы в $Z_{5}$ равен $-u / \varepsilon$.

Рассмотрим теперь диаграммы (28), чтобы проверить выполнение условия галилеевой инвариантности и для второй модели. Вычисление первой и третьей диаграмм не содержит никаких трудностей, поэтому обратимся сразу ко второй диаграмме. Как и в первом случае, достаточно пропустить внешний импульс $p_{\|}$от "хвоста" $\varphi^{\prime}$ к "хвосту" $\varphi$. Тогда аналитическое выражение для диаграммы принимает вид

$$
\begin{aligned}
I_{7}=( & \left.-\lambda g f^{1 / 4} \mu^{\varepsilon / 2}\right)^{2} \int \frac{d \mathbf{k}_{\perp} d k_{\|} d \eta}{(2 \pi)^{d+1}} \frac{-2 i \lambda k_{\|}}{\eta^{2}+\lambda^{2}\left(k_{\perp}^{2}+f k_{\|}^{2}+\tau\right)} \times \\
& \times \frac{1}{-i \eta+\lambda\left(k_{\perp}^{2}+f k_{\|}^{2}+\tau\right)} \frac{1}{i \eta+\lambda\left[k_{\perp}^{2}+f\left(k_{\|}-p_{\|}\right)^{2}+\tau\right]} .
\end{aligned}
$$

Взяв интеграл по $\eta$ с помощью вычетов и сложив возникающие от двух полюсов вклады, после некоторых преобразований получаем

$$
I_{7}=-\frac{i g^{2} \mu^{\varepsilon}}{4(2 \pi)^{d} f^{1 / 2}} \int d \mathbf{q} q_{\|} \frac{2\left(q^{2}+\tau\right)+\tilde{p}_{\|}\left(\tilde{p}_{\|} / 2-q_{\|}\right)}{\left(q^{2}+\tau\right)^{2}\left[q^{2}-q_{\|} \tilde{p}_{\|}+\tilde{p}_{\|}^{2} / 2+\tau\right]^{2}}
$$

(все обозначения уже пояснялись выше). Нас интересует линейный по $\tilde{p}_{\|}$член. При $\tilde{p}_{\|}=0$ подынтегральное выражение конечно, поэтому разложим его в ряд по этому импульсу. В итоге остается вычислить следующий интеграл:

$$
I_{7}=-\frac{i g^{2} \mu^{\varepsilon} \tilde{p}_{\|}}{4(2 \pi)^{d} f^{1 / 2}} \int d \mathbf{q} \frac{3 q_{\|}^{2}}{\left(q^{2}+\tau\right)^{4}} .
$$

Заметим, что в этом интеграле единственный источник анизотропии - множитель $q_{\|}^{2}$, поэтому можно заменить его на $q^{2} / d$, сведя подынтегральное выражение к изотропному. Вычисляя полученный интеграл, находим, что

$$
\begin{aligned}
\int d \mathbf{q} \frac{q^{2}}{\left(q^{2}+\tau\right)^{4}} & =\int \frac{d \mathbf{q}}{\left(q^{2}+\tau\right)^{3}}-\tau \int \frac{d \mathbf{q}}{\left(q^{2}+\tau\right)^{4}}= \\
& =\pi^{d / 2} \tau^{d / 2-3}\left[\frac{\Gamma(3-d / 2)}{2}-\frac{\Gamma(4-d / 2)}{6}\right] .
\end{aligned}
$$

Второе слагаемое конечно, так что его можно отбросить. Окончательно получаем

$$
I_{7}=-\frac{3 i p_{\|} g^{2} \mu^{\varepsilon}}{4 d(2 \pi)^{d}} \int d \mathbf{q} \frac{q^{2}}{\left(q^{2}+\tau\right)^{4}}=-\frac{3 i p_{\|} g^{2} \mu^{\varepsilon}}{8 d(2 \pi)^{d}} \tau^{-\varepsilon / 2} \Gamma\left(\frac{\varepsilon}{2}\right) \pi^{d / 2} \simeq \frac{-i p_{\|} u}{4 \varepsilon} .
$$

3 Теоретическая и математическая физика, т. 167, № 1, 2011 г. 
Вычисление единственной оставшейся диаграммы в (29) не содержит никаких принципиально новых моментов.

Итак, выпишем окончательные формулы для констант ренормировки $Z$ для второй модели:

$$
\begin{array}{ll}
Z_{1}=Z_{6}=Z_{7}=1-\frac{u}{2 \varepsilon}, & Z_{2}=1-\frac{u}{3 \varepsilon}, \\
Z_{3}=1-\frac{u}{3 \varepsilon}-\frac{w}{\xi}, & Z_{4}=Z_{5}=1-\frac{2 u}{\varepsilon} .
\end{array}
$$

Видно, что вытекающее из галилеевой инвариантности равенство $Z_{1}=Z_{6}$ для второй модели также выполняется.

\section{5. УРАВНЕНИЕ РГ}

Уравнение РГ записывается для ренормированной корреляционной функции $G_{\mathrm{R}}=\langle\Phi \ldots \Phi\rangle_{\mathrm{R}}$, которая отличается от первоначальной (неренормированной) функции $G=\langle\Phi \ldots \Phi\rangle$ только нормировкой и выбором параметров и, следовательно, с равным правом может быть использована для анализа критического поведения. Соотношение $\mathcal{S}_{(1,2) \mathrm{R}}(\Phi, e, \mu)=\mathcal{S}_{(1,2)}\left(\Phi, e_{0}\right)$ между функционалами действия $(10),(11)$ и $(16),(17)$ приводит к соотношению между корреляционными функциями:

$$
G\left(e_{0}, \ldots\right)=Z_{\varphi}^{N_{\varphi}} Z_{\varphi^{\prime}}^{N_{\varphi^{\prime}}} G_{\mathrm{R}}(e, \mu, \ldots),
$$

где, как обычно, $N_{\varphi}$ и $N_{\varphi^{\prime}}$ - числа входящих в $G$ полей (напомним, что в силу галилеевой инвариантности поле скорости не ренормируется: $Z_{v}=1$ ); здесь $e_{0}=\left\{\lambda_{0}, \tau_{0}, f_{0}, w_{0}, u_{0}\right\}$ - набор первоначальных параметров и $e=\{\lambda, \tau, f, w, u\}-$ их ренормированные аналоги; многоточием обозначены остальные переменные (время, координата, импульс и т. д.).

Применим оператор $\widetilde{\mathcal{D}}_{\mu} \equiv \mu \partial_{\mu}$ при фиксированных переменных из набора $e_{0}$. Это дает основное дифференциальное уравнение РГ для функции $G_{\mathrm{R}}(e, \mu, \ldots)$ :

$$
\left(\mathcal{D}_{\mathrm{RG}}+N_{\varphi} \gamma_{\varphi}+N_{\varphi^{\prime}} \gamma_{\varphi^{\prime}}\right) G_{\mathrm{R}}(e, \mu, \ldots)=0
$$

где $\mathcal{D}_{\mathrm{RG}}$ - оператор $\widetilde{\mathcal{D}}_{\mu}$, выраженный в ренормированных переменных,

$$
\mathcal{D}_{\mathrm{RG}} \equiv \mathcal{D}_{\mu}+\beta_{u} \partial_{u}+\beta_{w} \partial_{w}-\gamma_{f} \mathcal{D}_{f}-\gamma_{\lambda} \mathcal{D}_{\lambda}-\gamma_{\tau} \mathcal{D}_{\tau}
$$

Здесь и далее $\mathcal{D}_{x} \equiv x \partial_{x}$ для любой переменной $x$ и $\gamma_{F} \equiv \mathcal{D}_{\mu} \ln Z_{F}$ - аномальная размерность для любой величины $F$. В свою очередь $\beta$-функции для двух безразмерных констант $u$ и $w$ имеют вид

$$
\beta_{u} \equiv \widetilde{\mathcal{D}}_{\mu} u=u\left(-\varepsilon-\gamma_{u}\right), \quad \beta_{w} \equiv \widetilde{\mathcal{D}}_{\mu} w=w\left(-\xi-\gamma_{w}\right)
$$

где вторые равенства следуют из определений и соотношений (18). Равенства (19) приводят к следующим соотношениям между аномальными размерностями для модели (16):

$$
\begin{array}{lll}
\gamma_{1}=\gamma_{\varphi^{\prime}}+\gamma_{\varphi}, & \gamma_{2}=\gamma_{\lambda}+\gamma_{1}, & \gamma_{3}=\gamma_{f}+\gamma_{2}, \\
\gamma_{4}=\gamma_{\tau}+\gamma_{2}, & \gamma_{5}=\gamma_{\lambda}+\gamma_{g}+\frac{\gamma_{f}}{4}+\gamma_{\varphi^{\prime}}+2 \gamma_{\varphi}, & \gamma_{6}=\gamma_{\varphi^{\prime}}+\gamma_{\varphi}
\end{array}
$$


В силу (21) имеем $\gamma_{\varphi^{\prime}}=\gamma_{\varphi}$. Кроме того, так как $u \propto g^{2}$, справедливо равенство $Z_{u}=Z_{g}^{2}$, а значит, $\gamma_{u}=2 \gamma_{g}$. В модели (17) появляется дополнительная аномальная размерность

$$
\gamma_{7}=\gamma_{\lambda}+2 \gamma_{\varphi^{\prime}}
$$

Теперь разрешим равенство (36) и соотношения (35) относительно РГ-функций, входящих в уравнение РГ. Для модели (16) с взаимодействием Грибова получаем

$$
\begin{array}{lll}
\gamma_{\varphi^{\prime}}=\gamma_{\varphi}=\frac{\gamma_{1}}{2}, & \gamma_{\lambda}=\gamma_{2}-\gamma_{1}, & \gamma_{f}=\gamma_{3}-\gamma_{2}, \\
\gamma_{\tau}=\gamma_{4}-\gamma_{2}, & \gamma_{g}=\gamma_{5}-\frac{3}{4} \gamma_{2}-\frac{\gamma_{3}}{4}-\frac{\gamma_{1}}{2}, & \gamma_{w}=\gamma_{1}-\gamma_{3} .
\end{array}
$$

Выпишем сразу явные однопетлевые выражения для указанных величин, используя соотношения (25) и приближенное равенство

$$
\gamma_{F}=\left(\beta_{u} \partial_{u}+\beta_{w} \partial_{w}\right) \ln Z_{F} \simeq-\left(\varepsilon \mathcal{D}_{u}+\xi \mathcal{D}_{w}\right) \ln Z_{F},
$$

верное в первом порядке по $\varepsilon$ и $\xi$ (при выводе этого выражения мы учли, что величины $Z$ зависят только от констант связи $u$ и $w)$. Имеем

$$
\begin{array}{ll}
\gamma_{1}=-\frac{u}{4}+O\left(u^{2}\right), & \gamma_{2}=-\frac{u}{8}+O\left(u^{2}\right), \quad \gamma_{3}=-\frac{u}{8}+w+O\left(u^{2}\right), \\
\gamma_{4}=-\frac{u}{2}+O\left(u^{2}\right), & \gamma_{5}=-u+O\left(u^{2}\right),
\end{array}
$$

где символ $O\left(u^{2}\right)$ обозначает все старшие вклады типа $u^{2}, w^{2}, u w$ и т. д. Из формул (37) тогда находим

$$
\begin{array}{lll}
\gamma_{\varphi^{\prime}}=\gamma_{\varphi}=-\frac{u}{8}+O\left(u^{2}\right), & \gamma_{\lambda}=\frac{u}{8}+O\left(u^{2}\right), & \gamma_{f}=w+O\left(u^{2}\right), \\
\gamma_{\tau}=-\frac{3 u}{8}+O\left(u^{2}\right), & \gamma_{g}=-\frac{3 u}{4}-\frac{w}{4}+O\left(u^{2}\right), & \gamma_{w}=-\frac{u}{8}-w+O\left(u^{2}\right) .
\end{array}
$$

Аналогичные выражения можно записать для модели (17):

$$
\begin{gathered}
\gamma_{\varphi^{\prime}}=\frac{1}{2}\left(\gamma_{7}+\gamma_{1}-\gamma_{2}\right), \quad \gamma_{\varphi}=\frac{1}{2}\left(-\gamma_{7}+\gamma_{1}+\gamma_{2}\right), \\
\gamma_{\lambda}=\gamma_{2}-\gamma_{1}, \quad \gamma_{f}=\gamma_{3}-\gamma_{2}, \quad \gamma_{\tau}=\gamma_{4}-\gamma_{2}, \\
\gamma_{g}=\frac{1}{2}\left(\gamma_{7}-\gamma_{1}-\frac{5}{2} \gamma_{2}-\frac{\gamma_{3}}{2}+2 \gamma_{5}\right), \quad \gamma_{w}=\gamma_{1}-\gamma_{3} .
\end{gathered}
$$

В однопетлевом приближении, используя (30), получаем

$$
\begin{array}{ll}
\gamma_{1}=\gamma_{7}=\frac{u}{2}+O\left(u^{2}\right), & \gamma_{2}=\frac{u}{3}+O\left(u^{2}\right), \\
\gamma_{3}=\frac{u}{3}+w+O\left(u^{2}\right), & \gamma_{4}=\gamma_{5}=2 u+O\left(u^{2}\right) .
\end{array}
$$

С учетом равенств (40) находим

$$
\begin{aligned}
& \gamma_{\varphi^{\prime}}=\frac{u}{3}+O\left(u^{2}\right), \quad \gamma_{\varphi}=\frac{u}{6}+O\left(u^{2}\right), \quad \gamma_{\lambda}=-\frac{u}{6}+O\left(u^{2}\right), \\
& \gamma_{f}=w+O\left(u^{2}\right), \quad \gamma_{\tau}=\frac{5}{3} u+O\left(u^{2}\right), \quad \gamma_{g}=\frac{3}{2} u-\frac{w}{4}+O\left(u^{2}\right), \\
& \gamma_{w}=\frac{u}{6}-w+O\left(u^{2}\right) .
\end{aligned}
$$




\section{6. НЕПОДВИЖНЫЕ ТОЧКИ}

Как известно, возможные скейлинговые режимы (типы асимптотического поведения корреляционных функций в ИК-области) ренормируемой модели связаны с ИК-устойчивыми неподвижными точками уравнений РГ.

В общем случае неподвижные точки определяются условием обращения в ноль всех $\beta$-функций. В наших моделях координаты $u_{*}, w_{*}$ неподвижных точек находятся из уравнений

$$
\beta_{u}\left(u_{*}, w_{*}\right)=0, \quad \beta_{w}\left(u_{*}, w_{*}\right)=0,
$$

где $\beta$-функции определены в (34). Тип неподвижной точки определяется матрицей $\Omega$ с элементами $\Omega_{i j}=\partial \beta_{i} / \partial g_{j}$, где $\left\{\beta_{1}, \beta_{2}\right\}=\left\{\beta_{u}, \beta_{w}\right\}$ - полный набор $\beta$-функций и $\left\{g_{1}, g_{2}\right\}=\{u, w\}-$ полный набор констант связи. Для ИК-устойчивой неподвижной точки матрица $\Omega$ положительно определена, т. е. вещественные части всех ее собственных чисел больше нуля.

Рассмотрим сначала модель (16). Из определения (34) и явных выражений (39) для аномальных размерностей мы получаем следующие явные выражения (в главном порядке) для $\beta$-функций:

$$
\beta_{u}=u\left(-\varepsilon+\frac{3 u}{2}+\frac{w}{2}\right), \quad \beta_{w}=w\left(-\xi+\frac{u}{8}+w\right) .
$$

Из равенств (43) и (44) мы можем найти четыре различные неподвижные точки.

1. Гауссова (свободная) неподвижная точка $u_{*}=w_{*}=0$; матрица $\Omega$ в этом случае имеет вид

$$
\Omega=\left(\begin{array}{cc}
-\varepsilon & 0 \\
0 & -\xi
\end{array}\right)
$$

2. Точка $u_{*}=0, w_{*}=\xi$; матрица $\Omega$ в этом случае имеет вид

$$
\Omega=\left(\begin{array}{cc}
-\varepsilon+\frac{\xi}{2} & 0 \\
\frac{\xi}{8} & \xi
\end{array}\right) .
$$

3. Точка $u_{*}=2 \varepsilon / 3, w_{*}=0$; матрица $\Omega$ в этом случае имеет вид

$$
\Omega=\left(\begin{array}{cc}
\varepsilon & \frac{\varepsilon}{3} \\
0 & -\xi+\frac{\varepsilon}{12}
\end{array}\right) .
$$

4. Точка $u_{*}=\frac{8}{23}(2 \varepsilon-\xi), w_{*}=\frac{2}{23}(12 \xi-\varepsilon)$; матрица $\Omega$ в этом случае имеет вид

$$
\Omega=\left(\begin{array}{cc}
\frac{12}{23}(2 \varepsilon-\xi) & \frac{4}{23}(-\xi+2 \varepsilon) \\
\frac{1}{23}(3 \xi-4 \varepsilon) & \frac{2}{23}(12 \xi-\varepsilon)
\end{array}\right) .
$$

Для трех первых точек собственные числа матриц $\Omega$ совпадают, очевидно, с ее диагональными элементами, а для последнего случая они равны

$$
\lambda_{ \pm}=\frac{11}{23} \varepsilon+\frac{6}{23} \xi \pm \frac{\sqrt{167 \varepsilon^{2}-443 \varepsilon \xi+312 \xi^{2}}}{23} .
$$


Выражение под корнем положительно для любых $\varepsilon, \xi$, поэтому собственные числа вещественны.

Обратимся к модели (17). Для $\beta$-функций имеем

$$
\beta_{u}=u\left(-\varepsilon-3 u+\frac{w}{2}\right), \quad \beta_{w}=w\left(-\xi-\frac{u}{6}+w\right) .
$$

Из соотношений (43) и (49) вновь можно найти четыре неподвижные точки.

1. Гауссова (свободная) неподвижная точка $u_{*}=w_{*}=0$; матрица $\Omega$ в этом случае имеет вид

$$
\Omega=\left(\begin{array}{cc}
-\varepsilon & 0 \\
0 & -\xi
\end{array}\right) \text {. }
$$

2. Точка $u_{*}=0, w_{*}=\xi$; матрица $\Omega$ в этом случае имеет вид

$$
\Omega=\left(\begin{array}{cc}
-\varepsilon+\frac{\xi}{2} & 0 \\
-\frac{\xi}{6} & \xi
\end{array}\right)
$$

3. Точка $u_{*}=-\varepsilon / 3, w_{*}=0$; матрица $\Omega$ в этом случае имеет вид

$$
\Omega=\left(\begin{array}{cc}
\varepsilon & -\frac{\varepsilon}{6} \\
0 & -\xi+\frac{\varepsilon}{18}
\end{array}\right) \text {. }
$$

4. Точка $u_{*}=\frac{6}{35}(-2 \varepsilon+\xi), w_{*}=\frac{2}{35}(18 \xi-\varepsilon)$; матрица $\Omega$ в этом случае имеет вид

$$
\Omega_{i j}=\left(\begin{array}{cc}
\frac{18}{35}(2 \varepsilon-\xi) & \frac{3}{35}(\xi-2 \varepsilon) \\
\frac{1}{105}(\varepsilon-18 \xi) & \frac{2}{35}(18 \xi-\varepsilon)
\end{array}\right) .
$$

Для трех первых точек собственные числа матриц $\Omega$ вновь совпадают с ее диагональными элементами, а для последнего случая они имеют вид

$$
\lambda_{ \pm}=\frac{17}{35} \varepsilon+\frac{9}{35} \xi \pm \frac{\sqrt{359 \varepsilon^{2}-989 \varepsilon \xi+711 \xi^{2}}}{35}
$$

и также являются вещественными при любых значениях $\varepsilon, \xi$.

Здесь необходимо сделать следующее важное замечание. В модели (17) для третьей и четвертой неподвижных точек, в которых величина $u_{*}$ отлична от нуля, она отрицательна. Тем самым в силу соотношения $u \propto g^{2}$ такая точка не реализуется в модели с вещественной константой связи $g$. Однако даже простая статическая модель с взаимодействием $g \varphi^{3}$ в таком случае является, по-видимому, неустойчивой, так как ее потенциал взаимодействия не ограничен снизу (функциональный интеграл "расходится"). Напротив, такая модель с чисто мнимой константой связи оказывается состоятельной и имеет физические приложения: она описывает критическое поведение вблизи так называемого края Янга-Ли (см., например, работу [14]) и, что важно для нас в данном случае, описывает универсальные свойства метастабильных состояний, возникающих при фазовых переходах первого рода [10]. Более 


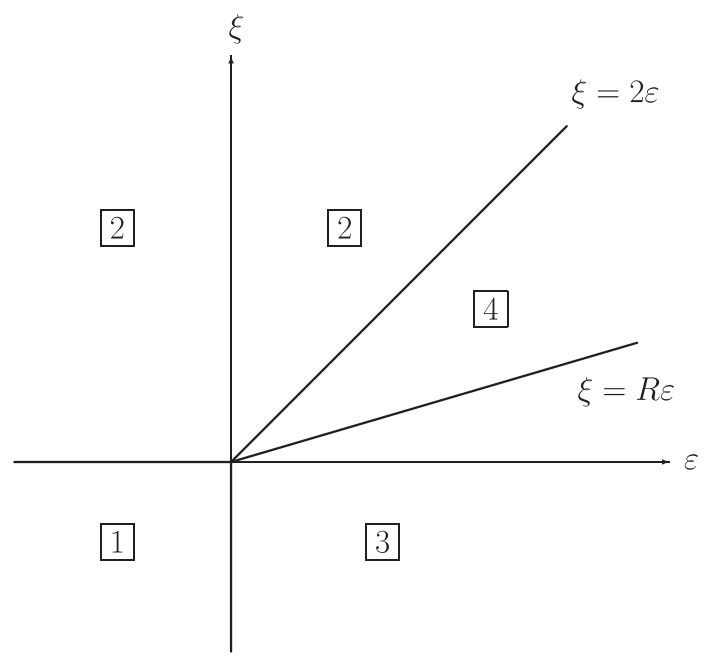

Области ИК-устойчивости неподвижных точек для моделей (16) и (17). В первом случае $\varepsilon=4-d$ и $R=1 / 12$, во втором $\varepsilon=6-d$ и $R=1 / 18$.

того, в псевдоевклидовом случае эта модель отвечает удовлетворительной модели квантовой теории поля (спектр энергии положителен и ограничен снизу) [15]. Перейти к такой модели можно с помощью подстановки $g \rightarrow i g$. При этом во всех константах $Z$ и функциях $\gamma$ и $\beta$ произойдет замена $u \rightarrow-u$, а величина $u_{*}$ станет положительной. Поскольку такая замена никак не отразится на областях устойчивости и критических размерностях, мы не будем выполнять ее в явном виде, а просто в дальнейшем будем считать константу взаимодействия $g$ чисто мнимой.

Первые неподвижные точки обеих моделей относятся к гауссову режиму (свободная теория поля), когда ни одно из взаимодействий не влияет на критическое поведение в главном приближении ИК-асимптотики (конечно, взаимодействия в моделях присутствуют, но они определяют лишь поправки к главным членам). Вторые точки отвечают критическому режиму, в котором нелинейности исходных моделей (взаимодействие Грибова и взаимодействие $\varphi^{\prime} \varphi^{2}$ второй модели) оказываются несущественными с точки зрения критического поведения, это случай пассивного скалярного поля без самодействия, претерпевающего лишь турбулентный перенос. Для третьих точек в том же смысле оказывается несущественным турбулентное перемешивание, т.е. критическое поведение для них такое же, как в исходных моделях без поля скорости. Четвертые, наиболее интересные, неподвижные точки в обеих моделях соответствуют новому скейлинговому ИК-режиму, в котором существенны обе нелинейности. Соответствующие критические размерности зависят от обоих параметров $\varepsilon, \xi$ уже в главном порядке и вычисляются как двойные ряды по этим параметрам.

Области ИК-устойчивости для этих неподвижных точек в плоскости $(\xi, \varepsilon)$ (т. е. области значений $\varepsilon$ и $\xi$, при которых собственные числа матрицы $\Omega$ для данной неподвижной точки положительны) для моделей (16) и (17) представлены на рисунке.

В главных приближениях (44) и (49) все границы областей устойчивости - просто прямые линии (границы этих областей, как уже было упомянуто, находятся из условия смены знака собственных чисел матрицы $\Omega$ ). Таким образом, в обеих 
моделях при любых $\varepsilon$ и $\xi$ мы попадаем в устойчивую область какой-то конкретной неподвижной точки; нет никаких "щелей" и перекрытий между различными областями (когда одновременно возникают несколько возможных скейлинговых режимов, выбор точки определяется начальными данными для уравнения РГ). В этом смысле критическое поведение универсально. Можно показать, что такая ситуация сохраняется и при учете старших порядков в $\beta$-функциях, хотя сами границы могут при этом искривляться. Это является следствием того факта, что у нас частные случаи $w=0$ или $u=0$ сами соответствуют замкнутым относительно ренормировки моделям, $\beta$-функции которых известны и имеют только одну гауссову и одну нетривиальную неподвижные точки.

Полученные результаты аналогичны выводам работ [8], где рассматривается тот же тип турбулентного перемешивания для критической динамики модели $\varphi^{4}$ (известной как модель $A$ ): характер неподвижных точек и области их устойчивости для всех трех случаев схожи. В частности, в однопетлевом приближении картина областей устойчивости для модели $A$ такая же, как на рисунке с $R=0$ и $\varepsilon=4-d$. Видно, что при изменении исходной (без турбулентного перемешивания) модели смещается только граница, разделяющая области 3 и $4(\xi=\varepsilon / 12, \xi=\varepsilon / 18$ в настоящей работе и $\xi=0$ в работах [8]). Это явление нетрудно объяснить. Во всех этих моделях $\beta$-функции имеют вид $\beta_{u}=u(-\varepsilon+a u+b w)$ и $\beta_{w}=w(-\xi+c u+d w)$ с некоторыми параметрами $a, b, c, d$. Можно убедиться, что граница между областями 2 и 4 определяется значениями параметров $b, d$, которые находятся из диаграмм, не включающих нелинейности по скалярным полям. Напротив, граница между областями 4 и 3 определяется параметрами $a, c$, которые находятся по диаграммам без участия поля скорости. Поэтому граница областей 2 и 4 определяется ансамблем поля скорости (и одинакова для всех трех упомянутых выше моделей), а граница областей 3 и 4 определяется исходной моделью без турбулентного переноса и для всех этих случаев различна. Скорее всего, в данном случае это свойства однопетлевого приближения.

\section{7. КРИТИЧЕСКИЙ СКЕЙЛИНГ И КРИТИЧЕСКИЕ РАЗМЕРНОСТИ}

Напомним определение обобщенной однородности. Пусть $F$ - некоторая функция от $n$ независимых аргументов $x_{1}, \ldots, x_{n}$, удовлетворяющая следующему размерному соотношению:

$$
F\left(\lambda^{\alpha_{1}} x_{1}, \ldots, \lambda^{\alpha_{n}} x_{n}\right)=\lambda^{\alpha_{F}} F\left(x_{1}, \ldots, x_{n}\right)
$$

с определенным набором постоянных коэффициентов (скейлинговых размерностей) $\left\{\alpha_{1}, \ldots, \alpha_{n}, \alpha_{F}\right\}$ и произвольным положительным параметром $\lambda>0$. Дифференцируя соотношение (55) по $\lambda$ и затем полагая $\lambda=1$, получаем дифференциальное уравнение первого порядка с постоянными коэффициентами

$$
\sum_{i=1}^{n} \alpha_{i} \mathcal{D}_{i} F\left(x_{1}, \ldots, x_{n}\right)=\alpha_{F} F\left(x_{1}, \ldots, x_{n}\right), \quad \mathcal{D}_{i}=x_{i} \frac{\partial}{\partial x_{i}},
$$

общее решение которого выглядит следующим образом:

$$
F\left(x_{1}, \ldots, x_{n}\right)=x_{1}^{\alpha_{F} / \alpha_{1}} \widetilde{F}\left(\frac{x_{2}}{x_{1}^{\alpha_{2} / \alpha_{1}}}, \ldots, \frac{x_{n}}{x_{1}^{\alpha_{n} / \alpha_{1}}}\right),
$$


где $\widetilde{F}$ - произвольная функция от $(n-1)$ аргумента. Очевидно, размерности определены с точностью до общего множителя (это можно увидеть, заменив $\lambda$ на $\lambda^{a}$ в (55) или умножив уравнение (56) на $a$ ); эта произвольность может быть устранена, например, если положить $\alpha_{1}=1$. Если $\alpha_{i}=0$ для какого-нибудь $x_{i}$, то эта переменная не преобразуется в (55), и соответствующая производная в (56) отсутствует.

Хорошо известно, что главный член, определяющий асимптотическое поведение (ренормированной) корреляционной функции на больших расстояниях, удовлетворяет уравнению РГ (32), в котором ренормированные константы связи заменены на их значения в неподвижных точках. В нашем случае это приводит к уравнению

$$
\left(D_{\mu}-\gamma_{f}^{*} D_{f}-\gamma_{\lambda}^{*} D_{\lambda}-\gamma_{\tau}^{*} D_{\tau}+\gamma_{\Phi}^{*} N_{\Phi}\right) G_{N_{\Phi}}=0,
$$

где $\gamma_{f}^{*}=\left.\gamma_{f}(g, w)\right|_{g=g_{*}, w=w_{*}}$ и аналогично для остальных $\gamma$, функция $G_{N_{\Phi}}-$ это ренормированная корреляционная функция с $N_{\Phi}=\left\{N_{\varphi^{\prime}}, N_{\varphi}, N_{v}\right\}$ полями (подразумевается суммирование по повторяющимся индексам).

Каноническая масштабная инвариантность функции $G_{N_{\Phi}}$ по отношению к трем независимым размерностям может быть выражена с помощью трех дифференциальных уравнений в форме (56). Действительно, из безразмерности действия вытекает его инвариантность относительно преобразования типа $\varphi(x) \rightarrow \lambda^{d \varphi} \varphi(\lambda x)$, а из этого вытекает свойство (55) для корреляционных функций и, следовательно, система дифференциальных уравнений.

Канонические размерности полей в наших двух моделях согласно таблицам 1 и 2 отличаются, поэтому выпишем указанные дифференциальные уравнения, не подставляя пока конкретные выражения:

$$
\begin{aligned}
\left(D_{\omega}+D_{\lambda}-d_{\Phi}^{\omega} N_{\Phi}\right) G_{N_{\Phi}} & =0, \\
\left(D_{\perp}+D_{\mu}-2 D_{\lambda}+2 D_{f}+2 D_{\tau}-d_{\Phi}^{\perp} N_{\Phi}\right) G_{N_{\Phi}} & =0, \\
\left(D_{\|}-2 D_{f}-d_{\Phi}^{\|} N_{\Phi}\right) G_{N_{\Phi}} & =0,
\end{aligned}
$$

где $D_{\|}=k_{\|} \partial / \partial k_{\|}, D_{\perp}=k_{\perp} \partial / \partial k_{\perp}$. Ясно, что уравнение (58) соответствует скейлинговому поведению типа (55) функции $G_{N_{\Phi}}$ при растягиваемых параметрах $\mu$, $\lambda, f$ и $\tau$ и при фиксированных импульсно-частотных переменных. Нас интересует критическое скейлинговое поведение, а именно поведение типа (55), в котором все ИК-существенные параметры (т. е. параметры, характеризующие близость к ИК-асимптотике: импульс/координата, время/частота, параметр $\tau$ ) меняются, в то время как ИК-несущественные параметры (т. е. параметры, остающиеся конечными в критической точке: $\lambda, \mu$ и $f$ ) фиксированы, см., например, [1]. Комбинируем уравнения (58), (59) так, что производные по ИК-несущественным параметрам исключатся; это приведет нас к желаемому уравнению, которое описывает критическое скейлинговое поведение:

$$
\left(D_{\perp}+\Delta_{\|} D_{\|}+\Delta_{\omega} D_{\omega}+\Delta_{\tau} D_{\tau}-N_{\Phi} \Delta_{\Phi}\right) G_{N_{\Phi}}=0
$$

здесь $\Delta_{\perp}=1$ - условие нормировки, а критическая размерность любого ИК-существенного параметра $F$ дается общим выражением

$$
\Delta_{F}=d_{F}^{\perp}+\Delta_{\|} d_{F}^{\|}+\Delta_{\omega} d_{F}^{\omega}+\gamma_{F}^{*}
$$


с каноническими размерностями из таблиц 1 и 2, причем верны соотношения

$$
\Delta_{\omega}=2-\gamma_{\lambda}^{*}, \quad \Delta_{\|}=\frac{2+\gamma_{f}^{*}}{2}
$$

Выпишем теперь окончательные однопетлевые результаты для критических размерностей. Для модели с взаимодействием Грибова (16) общие формулы (61) и (62) принимают вид

$$
\begin{gathered}
\Delta_{\omega}=2-\frac{u^{*}}{8}, \quad \Delta_{\|}=1+\frac{w^{*}}{2}, \\
\Delta_{\varphi^{\prime}}=\Delta_{\varphi}=\frac{d}{2}-\frac{u^{*}}{8}+\frac{w^{*}}{4}, \quad \Delta_{\tau}=2-\frac{3 u^{*}}{8} .
\end{gathered}
$$

Подставляя явные выражения для координат неподвижных точек, с учетом равенства $d=4-\varepsilon$ получаем для первой, второй, третьей, четвертой неподвижных точек соответственно

$$
\begin{gathered}
\Delta_{\omega}=2, \quad \Delta_{\|}=1, \quad \Delta_{\varphi^{\prime}}=\Delta_{\varphi}=\frac{d}{2}=2-\frac{\varepsilon}{2}, \quad \Delta_{\tau}=2 \\
\Delta_{\omega}=2, \quad \Delta_{\|}=1+\frac{\xi}{2}, \quad \Delta_{\varphi^{\prime}}=\Delta_{\varphi}=\frac{d}{2}+\frac{\xi}{4}=2-\frac{\varepsilon}{2}+\frac{\xi}{4}, \quad \Delta_{\tau}=2 \\
\Delta_{\omega}=2-\frac{\varepsilon}{12}, \quad \Delta_{\|}=1, \quad \Delta_{\varphi^{\prime}}=\Delta_{\varphi}=\frac{d}{2}-\frac{\varepsilon}{12}=2-\frac{7 \varepsilon}{12}, \quad \Delta_{\tau}=2-\frac{\varepsilon}{4} \\
\Delta_{\omega}=2-\frac{1}{23}(2 \varepsilon-\xi), \quad \Delta_{\|}=1+\frac{1}{23}(12 \xi-\varepsilon), \\
\Delta_{\varphi^{\prime}}=\Delta_{\varphi}=\frac{d}{2}+\frac{1}{46}(14 \xi-5 \varepsilon)=2+\frac{1}{23}(7 \xi-14 \varepsilon), \quad \Delta_{\tau}=2+\frac{3}{23}(\xi-2 \varepsilon) .
\end{gathered}
$$

Для модели (17) общие формулы (61) и (62) имеют вид

$$
\begin{gathered}
\Delta_{\omega}=2+\frac{u^{*}}{6}, \quad \Delta_{\|}=1+\frac{w^{*}}{2}, \quad \Delta_{\tau}=2+\frac{5 u^{*}}{3}, \\
\Delta_{\varphi^{\prime}}=\frac{d}{2}+1+\frac{u^{*}}{3}+\frac{w^{*}}{4}, \quad \Delta_{\varphi}=\frac{d}{2}-1+\frac{u^{*}}{6}+\frac{w^{*}}{4} .
\end{gathered}
$$

Подставляя явные выражения для координат неподвижных точек этой модели, получаем (теперь $d=6-\varepsilon$ ) для первой, второй, третьей, четвертой неподвижных точек соответственно

$$
\begin{gathered}
\Delta_{\omega}=2, \quad \Delta_{\|}=1, \quad \Delta_{\tau}=2, \quad \Delta_{\varphi^{\prime}}=\frac{d}{2}+1=4-\frac{\varepsilon}{2}, \\
\Delta_{\varphi}=\frac{d}{2}-1=2-\frac{\varepsilon}{2} ; \\
\Delta_{\omega}=2, \quad \Delta_{\|}=1+\frac{\xi}{2}, \quad \Delta_{\tau}=2, \\
\Delta_{\varphi^{\prime}}=\frac{d}{2}+1+\frac{\xi}{4}=4-\frac{\varepsilon}{2}+\frac{\xi}{4}, \quad \Delta_{\varphi}=\frac{d}{2}-1+\frac{\xi}{4}=2-\frac{\varepsilon}{2}+\frac{\xi}{4} ; \\
\Delta_{\omega}=2-\frac{\varepsilon}{18}, \quad \Delta_{\|}=1, \quad \Delta_{\tau}=2-\frac{5 \varepsilon}{9},
\end{gathered}
$$




$$
\begin{gathered}
\Delta_{\varphi^{\prime}}=\frac{d}{2}+1-\frac{\varepsilon}{9}=4-\frac{11 \varepsilon}{18}, \quad \Delta_{\varphi}=\frac{d}{2}-1-\frac{\varepsilon}{18}=2-\frac{5 \varepsilon}{9} \\
\Delta_{\omega}=2+\frac{1}{35}(\xi-2 \varepsilon), \quad \Delta_{\|}=1+\frac{1}{35}(18 \xi-\varepsilon), \quad \Delta_{\tau}=2+\frac{2}{7}(\xi-2 \varepsilon), \\
\Delta_{\varphi^{\prime}}=\frac{d}{2}+1+\frac{1}{70}(22 \xi-9 \varepsilon)=4+\frac{11}{35}(\xi-2 \varepsilon), \\
\Delta_{\varphi}=\frac{d}{2}-1+\frac{1}{14}(4 \xi-\varepsilon)=2+\frac{2}{7}(\xi-2 \varepsilon) .
\end{gathered}
$$

Для обеих моделей ответы для первых и вторых неподвижных точек точные. Прочие размерности имеют поправки - старшие степени по $\varepsilon$ для третьих неподвижных точек и старшие степени по $\varepsilon$ и $\xi$ для четвертых. Расчет этих поправок требует выхода за рамки однопетлевого приближения. Остается заметить, что в первом порядке критические размерности (64) и (66) на общих границах, разделяющих области устойчивости неподвижных точек, совпадают, т. е. оказываются непрерывными на этих прямых.

В качестве иллюстрации общих скейлинговых соотношений с размерностями (64) и (66) рассмотрим закон расплывания облака частиц, распространяющегося в критической среде "агента". Для простоты положим $\tau=0$, т. е. будем рассматривать непосредственно критическую точку.

В изотропном случае среднеквадратичный радиус облака выражается через функцию отклика следующим образом:

$$
R^{2}(t)=\int d^{d} \mathbf{x} x^{2}\left\langle\varphi^{\prime}(0, \mathbf{0}) \varphi(t, \mathbf{x})\right\rangle
$$

В нашем случае будем рассматривать аналоги этой величины для отдельных компонент:

$$
R_{i}^{2}(t)=\int d^{d-1} \mathbf{x}_{\perp} \int d x_{\|} x_{i}^{2}\left\langle\varphi^{\prime}(0,0, \mathbf{0}) \varphi\left(t, x_{\|}, \mathbf{x}_{\perp}\right)\right\rangle,
$$

где явно разделена зависимость от параллельных и перпендикулярных компонент вектора х. Подставим в последнее равенство вытекающее из формул предыдущих разделов асимптотическое скейлинговое представление для функции отклика при $\tau=0$ :

$$
\left\langle\varphi^{\prime}(0,0, \mathbf{0}) \varphi\left(t, x_{\|} t^{-1 / \Delta_{\omega}}, \mathbf{x}_{\perp}\right)\right\rangle=x_{\perp}^{-\Delta_{\varphi}-\Delta_{\varphi^{\prime}}} F\left(x_{\perp} t^{-1 / \Delta_{\omega}}, x_{\|}^{-\Delta_{\|} / \Delta_{\omega}}\right),
$$

где $x_{\perp}=\left|\mathbf{x}_{\perp}\right|$ и $F$ - некоторая функция от полностью безразмерных (в смысле критического скейлинга) аргументов. Перейдем к новым переменным $y_{\perp}=x_{\perp} t^{-1 / \Delta_{\omega}}$ и $y_{\|}=x_{\|} t^{-\Delta_{\|} / \Delta_{\omega}}$, так что вся зависимость от времени $t$ выделится из интегралов (67) в виде степенных множителей. При этом для перпендикулярной и параллельной компонент получаем $R_{\perp}^{2}(t) \propto t^{\alpha_{\perp}}$ и $R_{\|}^{2}(t) \propto t^{\alpha_{\|}}$с показателями степени

$$
\alpha_{\perp}=\frac{d+1+\Delta_{\|}-\Delta_{\varphi}-\Delta_{\varphi^{\prime}}}{\Delta_{\omega}}, \quad \alpha_{\|}=\frac{d-1+3 \Delta_{\|}-\Delta_{\varphi}-\Delta_{\varphi^{\prime}}}{\Delta_{\omega}} .
$$

Для изотропных режимов 1 и 3 (для обеих моделей) эти показатели совпадают, $\alpha_{\|}=\alpha_{\perp}$, причем для гауссовой точки мы получаем обычный закон диффузионного 
расплывания $R(t) \propto t^{1 / 2}$. Для режимов 2 и 4 расплывание облака оказывается анизотропным. В частности, для режима 2 (чисто турбулентный перенос пассивного скаляра) для обеих моделей получаем $\alpha_{\perp}=1$ и $\alpha_{\|}=1+\xi / 2$, т. е. в перпендикулярном "потоку" направлении происходит простая диффузия, $R_{\perp}^{2}(t) \propto t$, а в направлении "потока" расплывание ускоряется, $R_{\|}^{2}(t) \propto t^{1+\xi / 2}$. Последнее соотношение для значения $\xi=4 / 3$, отвечающего теории Колмогорова-Обухова, дает $R_{\|}^{2}(t) \propto t^{5 / 3}$, что можно представить также в виде $d R_{\|}^{2} / d t \propto R_{\|}^{4 / 5}$. Полученный “закон $4 / 5$ ” отличается от известного "закона $4 / 3$ Ричардсона" для изотропной турбулентности. Для режима 4 оба показателя (68) нетривиальны, не равны друг другу и зависят от обоих параметров ренормгруппового разложения по $\varepsilon$ и $\xi$.

\section{8. ЗАКЛЮЧЕНИЕ}

Мы рассмотрели критическое поведение двух динамических моделей: процесса Грибова и модели, описывающей фазовый переход первого рода в системе жидкостьпар. Поле скорости моделировалось гауссовой статистикой с исчезающим разновременным коррелятором и сильно анизотропной корреляционной функцией с асимптотическим поведением на бесконечности $\delta\left(t-t^{\prime}\right) / k_{\perp}^{d-1+\xi}$ (см. выражения (8), (9)).

Модели, изначально описываемые стохастическими дифференциальными уравнениями (1) и (3) с корреляторами шума (2) и (4), могут быть переформулированы в терминах мультипликативно-ренормируемых теорий поля, что позволяет применять метод РГ для изучения их критического поведения. Обе модели обнаруживают четыре разных ИК-асимптотических режима, определяемых четырьмя различными неподвижными точками уравнения РГ; их области устойчивости в координатах $(\varepsilon, \xi)$ найдены в главном порядке и отражены на рисунке. Эти режимы относятся к свободной неподвижной точке (режим 1), пассивному (нет обратного влияния на поле скорости) скалярному полю, при этом пренебрежимым становится член самодействия полей $\varphi$ и $\varphi^{\prime}$ (режим 2), равновесной критической динамике, в которой взаимодействие с полем скорости становится несущественным (режим 3), и наиболее нетривиальному сильно анизотропному режиму 4, в котором одинаково существенны оба взаимодействия.

Эти результаты получены, однако, в рамках однопетлевого приближения РГ, т. е. в главном порядке разложения по константам связи (для аномальных размерностей и $\beta$-функций) или параметрам отклонения от логарифмичности $\varepsilon$ и $\xi$ (для критических размерностей, неподвижных точек и областей их устойчивости). Возможность их экстраполяции на реальные конечные значения $\varepsilon$ и $\xi$ и достоверность полученных на их основе численных оценок для размерностей может вызывать сомнения, особенно для случая $\varepsilon=6-d$.

Серьезный анализ этих вопросов требует вычислений вкладов высших порядков РГ-разложений и применения к ним каких-либо процедур суммирования, как это было сделано, например, в работах [16] для статических моделей с кубичным взаимодействием типа $\varphi^{3}$. Подобный анализ выходит далеко за рамки данной работы, и мы надеемся выполнить его в дальнейшем.

Тем не менее можно уже сейчас привести некоторые аргументы в пользу сохранения описанной в разделе 6 картины неподвижных точек при реальных значениях 
параметров. А именно, при $u=0$ или $w=0$ наши модели сводятся к известным и относительно хорошо изученным моделям - анизотропной версии модели Обухова-Крейчнана [11] либо моделям критического поведения без учета движения среды. Поэтому положение неподвижных точек второго и третьего типов полностью определяется $\beta$-функциями этих моделей. При этом положение точек второго типа точно определяется в однопетлевом приближением (см., например, работу [13]). В свою очередь, положение точек третьего типа определяется $\beta$-функциями моделей из работ [2] и [16], которые известны в двух- и трехпетлевом приближении соответственно. Выполненный в указанных работах анализ подсказывает, что найденные в рамках $\varepsilon$-разложений точки "выживают" и при реальных $\varepsilon$. Тогда можно предположить, что и наиболее интересные для нас новые точки четвертого типа, лежащие в плоскости $(u, w)$ констант связи в квадранте, ограниченном точками $1-3$, также сохраняют свое положение и характер устойчивости при реальных значениях $\varepsilon$ и $\xi$.

Таким образом, мы можем заключить, что, используя наши упрощенные модели с гауссовым распределением скорости, уже в главном однопетлевом приближении можно получить информацию о наиболее важных характеристиках фазовых переходов в турбулентной среде: неустойчивость равновесного критического режима, появление новых неравновесных классов универсальности с новыми наборами критических показателей, достаточно сильно отличающихся от классических, и существование (для сильно анизотропного поля скорости) двух независимых масштабов длины.

Благодарности. Авторы благодарят за полезные обсуждения Л. Ц. Аджемяна, М. Гнатича и М. Ю. Налимова. Работа выполнена при финансовой поддержке РФФИ (грант № 08-02-00125_а) и Российской Национальной Программы (грант 2.1.1/1575). А. В. Малышев благодарит за поддержку фонд "Династия".

\section{Список литературы}

[1] А.Н. Васильев, Квантово-полевая ренормгруппа в теории критического поведения и стохастической динамике, Изд-во ПИЯФ, СПб., 1998.

[2] H.-K. Janssen, U. C. Täuber, Ann. Phys., 315:1 (2004), 147-192, arXiv: cond-mat/0409670.

[3] Д. Ю. Иванов, Критическое поведение неидеализированных систем, Физматлит, М., 2003.

[4] A. Onuki, K. Kawasaki, Progr. Theor. Phys., 63:1 (1980), 122-139; T. Imaeda, A. Onuki, K. Kawasaki, Progr. Theor. Phys., 71:1 (1984), 16-26.

[5] D. Beysens, M. Gbadamassi, L. Boyer, Phys. Rev. Lett., 43:17 (1979), 1253-1256; D. Beysens, M. Gbadamassi, J. Phys. Lett., 40:21 (1979), 565-567.

[6] R. Ruiz, D. R. Nelson, Phys. Rev. A, 23:6 (1981), 3224-3246; 24:5 (1981), 2727-2734; J. A. Aronowitz, D. R. Nelson, Phys. Rev. A, 29:4 (1984), 2012-2016.

[7] N. V. Antonov, M. Hnatich, J. Honkonen, J. Phys. A, 39:25 (2006), 7867-7887, arXiv: cond-mat/0604434.

[8] N. V. Antonov, A. A. Ignatieva, J. Phys. A, 39:44 (2006), 13593-13620, arXiv: cond-mat/0607019; N. V. Antonov, A. A. Ignatieva, A. V. Malyshev, Phys. Part. Nucl., 41:7 (2010), 998-1000, arXiv: 1003.2855.

[9] N. V. Antonov, V.I. Iglovikov, A.S. Kapustin, J. Phys. A, 42:13 (2009), 135001, 19 pp., arXiv: 0808.0076; N. V. Antonov, A. S. Kapustin, J. Phys. A, 43:40 (2010), 405001, 22 pp., arXiv: 1006.3133 .

[10] F. Zhong, Q. Chen, Phys. Rev. Lett., 95:17 (2005), 175701, 4 pp. 
[11] M. Avellaneda, A. J. Majda, Comm. Math. Phys., 131:2 (1990), 381-429; 146:1 (1992), 139-204.

[12] G. Falkovich, K. Gawȩdzki, M. Vergassola, Rev. Modern Phys., 73:4 (2001), 913-975, arXiv: cond-mat/0105199.

[13] N. V. Antonov, J. Phys. A, 39:25 (2006), 7825-7865.

[14] M. E. Fisher, Phys. Rev. Lett., 40:25 (1978), 1610-1613.

[15] C. M. Bender, D. C. Brody, H. F. Jones, Phys. Rev. Lett., 93:25 (2004), 251601, 4 pp., arXiv: hep-th/0402011.

[16] O.F. de Alcantara Bonfim, J.E. Kirkham, A. J. McKane, J. Phys. A, 13:7 (1980), L247-L251; 14:9 (1981), 2391-2413.

Поступила в редакцию 16.06.2010, после доработки 15.10.2010 